\title{
Sharp maximal functions associated with approximations of the identity in spaces of homogeneous type and applications
}

by

\author{
José María Martell (Madrid)
}

\begin{abstract}
In the context of the spaces of homogeneous type, given a family of operators that look like approximations of the identity, new sharp maximal functions are considered. We prove a good- $\lambda$ inequality for Muckenhoupt weights, which leads to an analog of the Fefferman-Stein estimate for the classical sharp maximal function. As a consequence, we establish weighted norm estimates for certain singular integrals, defined on irregular domains, with Hörmander conditions replaced by some estimates which do not involve the regularity of the kernel. We apply these results to prove the boundedness of holomorphic functional calculi on Lebesgue spaces with Muckenhoupt weights. In particular, some applications are given to second order elliptic operators with different boundary conditions.
\end{abstract}

1. Introduction. Let us consider a space of homogeneous type $(\mathcal{X}, d, \mu)$ which is a set $\mathcal{X}$ endowed with a distance $d$ and a non-negative Borel measure $\mu$ on $\mathcal{X}$ such that the doubling condition

$$
\mu(B(x, 2 r)) \leq C \mu(B(x, r))<\infty
$$

holds for all $x \in \mathcal{X}$ and $r>0$, where $B(x, r)=\{y \in \mathcal{X}: d(x, y)<r\}$. A more general definition and further studies of these spaces can be found in [CW], [MS1], [MS2].

Throughout this paper we will use the following notation: for every ball $B, x_{B}$ and $r_{B}$ are respectively its center and its radius, that is, $B=$ $B\left(x_{B}, r_{B}\right)$. Given $\lambda>0$, we will write $\lambda B$ for the $\lambda$-dilated ball, which is the ball with the same center as $B$ and with radius $r_{\lambda B}=\lambda r_{B}$. By $L_{0}^{p}(\mathcal{X})$, $1 \leq p \leq \infty$, we denote the set of functions in $L^{p}(\mathcal{X})$ with bounded support.

If $C$ is the smallest constant for which the measure $\mu$ satisfies the doubling condition (1), then $D=\log _{2} C$ is called the doubling order of $\mu$ and

2000 Mathematics Subject Classification: 42B25, 42B20, 47G10, 47A60, 47F05.

Key words and phrases: singular integrals, Muckenhoupt weights, sharp maximal functions, spaces of homogeneous type, holomorphic calculi, semigroup kernels, elliptic operators.

The author is partially supported by MCYT grant BFM2001-0189. 
we have

$$
\frac{\mu(B)}{\mu(\widetilde{B})} \leq C_{\mu}\left(\frac{r_{B}}{r_{\widetilde{B}}}\right)^{D} \quad \text { for all balls } \widetilde{B} \subset B \subset \mathcal{X} .
$$

In particular, it follows that $\mu(\lambda B) \leq C_{\mu} \lambda^{D} \mu(B)$ for every ball $B$ and for every $\lambda \geq 1$. Moreover, there exist $c_{\mu} \geq 1$ and $0 \leq N \leq D$ such that

$$
\mu(B(x, r)) \leq c_{\mu}\left(1+\frac{d(x, y)}{r}\right)^{N} \mu(B(y, r))
$$

uniformly on $x, y \in \mathcal{X}$ and $r>0$. In fact, this estimate with $N=D$ is a trivial consequence of (2) and the triangular inequality for the distance $d$. But, in general, $N$ could be smaller. This is, for example, the case of the Lebesgue measure on $\mathbb{R}^{d}$ or the case of Lie groups with polynomial growth, where (3) holds with $N=0$. On the other hand, let us point out that the doubling condition implies that $\mathcal{X}$ is bounded if and only if $\mu(\mathcal{X})<\infty$.

We consider the following "approximations of the identity" which previously appeared in $[\mathrm{DM}]$.

Definition 1.1. A family of operators $\left\{D_{t}: t>0\right\}$ is said to be an "approximation of the identity" if, for every $t>0, D_{t}$ is represented by the kernel $a_{t}(x, y)$, which is a measurable function defined on $\mathcal{X} \times \mathcal{X}$, in the following sense: for every $f \in L^{p}(\mathcal{X}), p \geq 1$,

$$
D_{t} f(x)=\int_{\mathcal{X}} a_{t}(x, y) f(y) d \mu(y)
$$

and the following condition holds:

$$
\begin{aligned}
\left|a_{t}(x, y)\right| \leq h_{t}(x, y)=\frac{1}{\mu\left(B\left(x, t^{1 / m}\right)\right)} s\left(d(x, y)^{m} t^{-1}\right), & \\
& (x, y) \in \mathcal{X} \times \mathcal{X}, t>0,
\end{aligned}
$$

where $m$ is a positive fixed constant and $s$ is a positive, bounded, decreasing function satisfying

$$
\lim _{r \rightarrow \infty} r^{D+\vartheta} s\left(r^{m}\right)=0,
$$

for some $\vartheta>N$ (recall that $N$ is the power appearing in (3) and $D$ is the doubling order of $\mu$ ).

The idea is that the kernels are controlled by positive decreasing radial functions which decay to 0 at infinity fast enough. Some examples of these functions $h_{t}$ are given by Gaussian or Poisson kernels. Associated with an "approximation of the identity" we next define a new sharp maximal function.

Definition 1.2. Given $f \in L^{p}(\mathcal{X})$ for some $p \geq 1$, we define the sharp maximal function associated with the "approximation of the identity" $\left\{D_{t}\right.$ : 
$t>0\}$ as

$$
\mathcal{M}_{D}^{\sharp} f(x)=\sup _{B \ni x} \frac{1}{\mu(B)} \int_{B}\left|f(y)-D_{t_{B}} f(y)\right| d \mu(y),
$$

where $t_{B}=r_{B}^{m}$.

In comparison with the classical Fefferman-Stein sharp maximal function $\mathcal{M}^{\sharp}$, we see that instead of subtracting from $f$ its average on every ball, we subtract $D_{t_{B}} f$. We will show that in case $D_{t} 1=1$ a.e., our sharp maximal function $\mathcal{M}_{D}^{\sharp}$ is pointwise smaller than $\mathcal{M}^{\sharp}$ but they are not comparable in general.

After some preliminaries, in Section 3 we state some properties of $\mathcal{M}_{D}^{\sharp}$. In Section 4 we prove a good- $\lambda$ inequality for this new sharp maximal function and the Hardy-Littlewood maximal operator $\mathcal{M}$. This estimate implies that $\mathcal{M}$ is controlled by $\mathcal{M}_{D}^{\sharp}$ in the $L^{p}(w)$ norm for $0<p<\infty$ and for every Muckenhoupt weight $w$ in the class $\mathcal{A}_{\infty}$ (see the definition in the next section). That is, we obtain an analog of the classical inequality of Fefferman-Stein in [FS] (for the weighted case see also [CF]) for this new sharp maximal function. In Section 5 we apply this result to some singular integrals introduced in $[\mathrm{DM}]$. For these operators the classical Hörmander type inequalities are replaced by some conditions that involve the "approximations of the identity" defined above. The advantage is that there is no regularity assumption, and this fact allows us to consider operators defined on non-smooth domains (see Section 6). We prove that such an operator $T$ is of strong type $(p, p), 1<p<\infty$, with respect to $w \in \mathcal{A}_{p}$. Here, the key is the control of $\mathcal{M}$ by $\mathcal{M}_{D}^{\sharp}$ obtained with the good- $\lambda$ inequality plus the fact that

$$
\mathcal{M}_{D}^{\sharp}(T f)(x) \leq C\left(M\left(|f|^{s}\right)(x)\right)^{1 / s}
$$

holds for every $1<s<\infty$. Let us point out the analogy between this estimate and the one holding for classical Calderón-Zygmund operators (see [GR, Chapter II, Theorem 5.20] for instance). For $p=1$, by means of the Calderón-Zygmund decomposition, we deduce that $T$ is of weak type $(1,1)$ with respect to weights in $\mathcal{A}_{1}$. In Section 7 we consider holomorphic functional calculi of elliptic operators, proving that they satisfy weighted estimates for Muckenhoupt weights. As a consequence, weighted estimates are studied for some second order elliptic operators with different boundary conditions.

The results contained in this paper are part of the author's Ph.D. thesis, written under the supervision of Prof. J. García-Cuerva (see [Mar]). The study of weighted estimates for the class of operators considered here was motivated by $[\mathrm{DM}]$, where the unweighted case is treated. During the preparation of this paper, I became aware of the paper [DY], where the authors 
prove weighted $L^{p}$ estimates of a different kind for holomorphic functional calculi of linear elliptic operators. They consider two weights, one on each side, and study conditions on the weight on one side which guarantee the existence of another weight on the other side. Their approach is based on the technique, already used in $[\mathrm{GM}]$ and $[\mathrm{GR}]$, of proving vector-valued inequalities for those operators.

The author would like to thank Prof. J. García-Cuerva for his encouragement, guidance, and for many useful discussions about the material of this article. The author would like to express his gratitude to Prof. S. Hofmann for his comments and suggestions.

2. Preliminaries. The Hardy-Littlewood maximal function is defined by

$$
\mathcal{M} f(x)=\sup _{B \ni x} \frac{1}{\mu(B)} \int_{B}|f(y)| d \mu(y) .
$$

We will also consider the centered version of this maximal operator, denoted by $\mathcal{M}^{\mathrm{c}}$, where the supremum is only taken over all balls centered at $x$. By the doubling condition, these maximal operators satisfy

$$
\mathcal{M}^{\mathrm{c}} f(x) \leq \mathcal{M} f(x) \leq C_{\mu} 2^{D} \mathcal{M}^{\mathrm{c}} f(x) .
$$

Note that $h_{t}$ in Definition 1.1 might be non-symmetric, however just by using (3),

$h_{t}(x, y) \leq c_{\mu} \min \left\{\frac{1}{\mu\left(B\left(x, t^{1 / m}\right)\right)}, \frac{1}{\mu\left(B\left(y, t^{1 / m}\right)\right)}\right\} s_{1}\left(d(x, y)^{m} t^{-1}\right)=\widetilde{h}_{t}(x, y)$,

where $s_{1}$ is a new function satisfying the same properties as $s$ with some $\vartheta>0$ and $\widetilde{h}_{t}(x, y)$ is symmetric. We also have

$$
C^{-1} \leq \int_{\mathcal{X}} h_{t}(x, y) d \mu(x) \leq C \quad \text { and } \quad C^{-1} \leq \int_{\mathcal{X}} h_{t}(y, x) d \mu(x) \leq C
$$

uniformly on $y \in \mathcal{X}, t>0$. On the other hand, as in [DR, p. 97], it is verified

$$
\left|D_{t} f(x)\right| \leq \int_{\mathcal{X}} h_{t}(x, y)|f(y)| d \mu(y) \leq \int_{\mathcal{X}} \widetilde{h}_{t}(x, y)|f(y)| d \mu(y) \leq C \mathcal{M} f(x),
$$

for every $f \in L^{p}(\mathcal{X}), 1 \leq p \leq \infty$. We also point out that

$$
\int_{\mathcal{X}} h_{t}(x, y)|f(x)| d \mu(x) \leq \int_{\mathcal{X}} \widetilde{h}_{t}(y, x)|f(x)| d \mu(x) \leq C \mathcal{M} f(y),
$$

where we have used the symmetry $\widetilde{h}_{t}(x, y)$. In both cases, these estimates are uniform on $t>0$. 
We consider Muckenhoupt weights. Given $1<p<\infty$, a weight $w$ belongs to $\mathcal{A}_{p}$ if, for every ball $B \subset \mathcal{X}$, it verifies

$$
\left(\mathcal{A}_{p}\right) \quad\left(\frac{1}{\mu(B)} \int_{B} w(x) d \mu(x)\right)\left(\frac{1}{\mu(B)} \int_{B} w(x)^{1-p^{\prime}} d \mu(x)\right)^{p-1} \leq C .
$$

The class $\mathcal{A}_{1}$ is defined by letting $p \rightarrow 1$, that is,

$$
\left(\frac{1}{\mu(B)} \int_{B} w(x) d \mu(x)\right)\left\|w^{-1}\right\|_{L^{\infty}(B)} \leq C,
$$

where the constant $C$ does not depend on the ball $B$. Finally, $\mathcal{A}_{\infty}$ is the union of the $\mathcal{A}_{p}$ classes, $1 \leq p<\infty$. These classes, in the Euclidean setting, were introduced by Muckenhoupt in [Muc] and further developed in [CF]. About Muckenhoupt weights in spaces of homogeneous type the reader is referred to $[\mathrm{ST}]$.

For $w \in \mathcal{A}_{\infty}$ and for every measurable set $E \subset \mathcal{X}$ we use the standard notation $w(E)=\int_{E} w(x) d \mu(x)$. Moreover, if $0<p<\infty$, then $L^{p}(\mathcal{X}, w)=$ $L^{p}(\mathcal{X}, w d \mu)$. In some parts of this work, we will be working in $\Omega \subset \mathcal{X}$. In that case, $L^{p}(\Omega)=L^{p}(\Omega, \mu)$ and, for $w \in \mathcal{A}_{\infty}(\mathcal{X})$, we will write $L^{p}(\Omega, w)=$ $L^{p}(\Omega, w d \mu)$. When we consider $w \in \mathcal{A}_{\infty}(\mathcal{X})$, we mean that $w$ is a Muckenhoupt weight with respect to the whole space $\mathcal{X}$ and not with respect to $\Omega$. We will simply use the notation $w \in \mathcal{A}_{\infty}$, omitting $\mathcal{X}$, when it is clear from the context.

The following result contains the Calderón-Zygmund decomposition on spaces of homogeneous type.

Theorem 2.1 ([CW]). Let $f \geq 0, f \in L_{0}^{1}(\mathcal{X})$, and $\lambda>\|f\|_{L^{1}(\mathcal{X})}(\mu(\mathcal{X}))^{-1}$. Then there exists a family of balls $\left\{B_{i}\right\}$ such that:

(i) $f(x) \leq C \lambda$ for $\mu$-a.e. $x \in \mathcal{X} \backslash \bigcup_{i} B_{i}$.

(ii) $\frac{1}{\mu\left(B_{i}\right)} \int_{B_{i}} f(y) d \mu(y) \leq C \lambda$.

(iii) $\sum_{i} \mu\left(B_{i}\right) \leq \frac{C}{\lambda} \int_{\mathcal{X}} f(y) d \mu(y)$.

(iv) There exists an integer $M \geq 1$, independent of $f$ and $\lambda$, such that every point in $\mathcal{X}$ belongs to at most $M$ of these balls.

REMARK 2.2. The following properties are contained in the proof of the previous result:

(i) There exists some constant $C_{\mathcal{X}}$, which only depends on the space, such that

$$
E_{\lambda}=\left\{x \in \mathcal{X}: \mathcal{M} f(x)>\lambda C_{\mathcal{X}}\right\}=\bigcup_{i} B_{i} .
$$

(ii) There exists $\varepsilon_{0}>1$, independent of $f$ and $\lambda$, such that $\left(\varepsilon_{0} B_{i}\right) \backslash E_{\lambda} \neq \emptyset$. 
3. A new sharp maximal function. In what follows $\left\{D_{t}: t>0\right\}$ will be an "approximation of the identity". We will use the following notation: if $B$ is a ball, $t_{B}=r_{B}^{m}$. We are going to study some properties of the maximal operator $\mathcal{M}_{D}^{\sharp}$ (see Definition 1.2).

Let us recall that the Fefferman-Stein sharp maximal function is defined as

$$
\mathcal{M}^{\sharp} f(x)=\sup _{B \ni x} \frac{1}{\mu(B)} \int_{B}\left|f(y)-f_{B}\right| d \mu(y),
$$

where $f_{B}$ stands for the $\mu$-average of $f$ over $B$.

Proposition 3.1. Let $\left\{D_{t}: t>0\right\}$ be an "approximation of the identity" such that for every $t>0, D_{t} 1=1$ a.e. or, what is the same, for every $t>0$,

$$
\int_{\mathcal{X}} a_{t}(x, y) d \mu(y)=1 \quad \text { for almost all } x \in \mathcal{X} .
$$

Then, for every function $f \in L^{p}(\mathcal{X}), p \geq 1$, and $x \in \mathcal{X}$ we have

$$
\mathcal{M}_{D}^{\sharp} f(x) \leq C \mathcal{M}^{\sharp} f(x) .
$$

However, the converse inequality does not hold in general.

REMARK 3.2. If the kernels of $D_{t}, t>0$, have bounded support, then the previous inequality is still true for locally integrable functions since the operators $D_{t}$ act over them.

REMARK 3.3. The condition $D_{t} 1=1$ a.e. is necessary for (8). This is obtained by taking $f(x)=1$. Then (8) implies that $\mathcal{M}_{D}^{\sharp} f=0$ and thus, for every $t>0, D_{t} 1=1$ outside of a zero measure set.

Proof of Proposition 3.1. We fix $f \in L^{p}(\mathcal{X})(p \geq 1), x_{0} \in \mathcal{X}$ and a ball $B \ni x_{0}$. Then

$$
\begin{aligned}
& \frac{1}{\mu(B)} \int_{B}\left|f(x)-D_{t_{B}} f(x)\right| d \mu(x) \\
& \leq \frac{1}{\mu(B)} \iint_{B} h_{t_{B}}(x, y)|f(x)-f(y)| d \mu(y) d \mu(x) \\
& \quad=\frac{1}{\mu(B)} \int_{B} \int_{2 B} \ldots d \mu(y) d \mu(x)+\sum_{k=1}^{\infty} \frac{1}{\mu(B)} \int_{B} \int_{2^{k+1} B \backslash 2^{k} B} \ldots d \mu(y) d \mu(x) \\
& \quad=I+I I .
\end{aligned}
$$

We estimate $I$. By (3) we have $\mu(B) \leq 2^{N} c_{\mu} \mu\left(B\left(x, r_{B}\right)\right)$ since $x \in B$. Moreover, for $y \in 2 B$ we get

$$
h_{t_{B}}(x, y)=\frac{s\left(d(x, y)^{m} t_{B}^{-1}\right)}{\mu\left(B\left(x, t_{B}^{1 / m}\right)\right)} \leq \frac{s(0)}{\mu\left(B\left(x, r_{B}\right)\right)} \leq \frac{C}{\mu(B)} \leq \frac{C}{\mu(2 B)},
$$


where in the first inequality we have used the fact that $s$ is decreasing. In this way, we obtain:

$$
I \leq \frac{C}{\mu(B) \mu(2 B)} \int_{B} \int_{2 B}|f(x)-f(y)| d \mu(y) d \mu(x) \leq C \mathcal{M}^{\sharp} f\left(x_{0}\right),
$$

since $x_{0} \in B \subset 2 B$. Regarding $I I$, if $x \in B$ and $y \in 2^{k+1} B \backslash 2^{k} B$ then $d(x, y) \geq 2^{k-1} r_{B}$ and, just as before,

$$
h_{t_{B}}(x, y)=\frac{s\left(d(x, y)^{m} r_{B}^{-m}\right)}{\mu\left(B\left(x, r_{B}\right)\right)} \leq C \frac{s\left(2^{(k-1) m}\right)}{\mu(B)} \leq C \frac{s\left(2^{(k-1) m}\right) 2^{(k+1) D}}{\mu\left(2^{k+1} B\right)},
$$

where we used (2). Thus,

$$
I I \leq C \sum_{k=1}^{\infty} 2^{k D} s\left(2^{(k-1) m}\right) \frac{1}{\mu(B) \mu\left(2^{k+1} B\right)} \int_{B} \int_{2^{k+1} B}|f(x)-f(y)| d \mu(y) d \mu(x) .
$$

We estimate each term as follows:

$$
\begin{aligned}
& \frac{1}{\mu(B) \mu\left(2^{k+1} B\right)} \int_{B} \int_{2^{k+1} B}|f(x)-f(y)| d \mu(y) d \mu(x) \\
& \leq \frac{1}{\mu\left(2^{k+1} B\right)} \int_{2^{k+1} B}\left|f(y)-f_{2^{k+1} B}\right| d \mu(y)+\frac{1}{\mu(B)} \int_{B}\left|f(x)-f_{2^{k+1} B}\right| d \mu(x) \\
& \leq \mathcal{M}^{\sharp} f\left(x_{0}\right)+\frac{1}{\mu(B)} \int_{B}\left|f(x)-f_{B}\right| d \mu(x)+\left|f_{B}-f_{2 B}\right|+\ldots+\left|f_{2^{k} B}-f_{2^{k+1} B}\right| \\
& \leq C \mathcal{M}^{\sharp} f\left(x_{0}\right)(k+1) .
\end{aligned}
$$

Therefore, by (4) we have

$$
I I \leq C \mathcal{M}^{\sharp} f\left(x_{0}\right) \sum_{k=1}^{\infty}(k+1) 2^{k D} s\left(2^{(k-1) m}\right) \leq C \mathcal{M}^{\sharp} f\left(x_{0}\right) .
$$

Next we are going to show why the converse inequality does not hold in general. To do that we consider $\mathbb{R}$ with the Lebesgue measure $d x$ and the "approximation of the identity" $\left\{D_{t}: t>0\right\}$ given by the kernels

$$
\begin{aligned}
a_{t}(x, y) & =\frac{1}{\left|\left(y-t^{1 / m}, y+t^{1 / m}\right)\right|} \chi_{\left(y-t^{1 / m}, y+t^{1 / m}\right)}(x) \\
& =\frac{1}{2 t^{1 / m}} \chi_{\left(x-t^{1 / m}, x+t^{1 / m}\right)}(y) .
\end{aligned}
$$

As we observed in Remark 3.2, since the supports of these kernels are bounded sets, the operators $D_{t}$ act over locally integrable functions. Let us take the function $f(x)=x$. Then, for every $t>0, D_{t} f(x)=x$ and thus $\mathcal{M}_{D}^{\sharp} f(x)=0$. On the other hand, $\mathcal{M}^{\sharp} f(x)=\infty$, and it is clear that $\mathcal{M}^{\sharp}$ and $\mathcal{M}_{D}^{\sharp}$ are not comparable. 
Next, we are going to show some properties of our sharp maximal function $\mathcal{M}_{D}^{\sharp}$ to be used later.

Lemma 3.4. For $1 \leq p \leq \infty$ and $f \in L^{p}(\mathcal{X})$, it follows that for every $x \in \mathcal{X}$,

$$
\left|D_{t} f(x)\right| \leq \frac{C}{\mu\left(B\left(x, t^{1 / m}\right)\right)^{1 / p}}\|f\|_{L^{p}(\mathcal{X})} .
$$

Proof. The case $p=1$ works because $s$ is a bounded function. For $p=\infty$, the properties of $h_{t}$ yield

$$
\left|D_{t} f(x)\right| \leq\|f\|_{L^{\infty}(\mathcal{X})} \int_{\mathcal{X}} h_{t}(x, y) d \mu(y) \leq C\|f\|_{L^{\infty}(\mathcal{X})} .
$$

Finally, if $1<p<\infty$, we combine Hölder's inequality and the former ideas to get

$$
\begin{aligned}
\left|D_{t} f(x)\right| & \leq\|f\|_{L^{p}(\mathcal{X})}\left(\int_{\mathcal{X}} h_{t}(x, y)^{p^{\prime}} d \mu(y)\right)^{1 / p^{\prime}} \\
& =\|f\|_{L^{p}(\mathcal{X})}\left(\int_{\mathcal{X}} h_{t}(x, y)^{p^{\prime}-1} h_{t}(x, y) d \mu(y)\right)^{1 / p^{\prime}} \\
& \leq\|f\|_{L^{p}(\mathcal{X})} \frac{C}{\mu\left(B\left(x, t^{1 / m}\right)\right)^{\left(p^{\prime}-1\right) / p^{\prime}}}\left(\int_{\mathcal{X}} h_{t}(x, y) d \mu(y)\right)^{1 / p^{\prime}} \\
& \leq\|f\|_{L^{p}(\mathcal{X})} \frac{C}{\mu\left(B\left(x, t^{1 / m}\right)\right)^{1 / p}} .
\end{aligned}
$$

Lemma 3.5. Consider $f \in L^{p}(\mathcal{X})$ for some $p \geq 1$, a ball $B$ and $x \in B$. Then there exists $C>0$ (independent of $f, B, x)$ such that

$$
\frac{1}{\mu(B)} \int_{B}\left|D_{t_{B}} f(y)\right| d \mu(y) \leq C \mathcal{M} f(x) .
$$

Proof. First of all,

$$
\left|D_{t_{B}}\left(f \chi_{4 B}\right)(y)\right| \leq \sup _{z \in 4 B} h_{t_{B}}(y, z) \mu(4 B) \mathcal{M} f(x),
$$

since $x \in B \subset 4 B$. By [DR, Proposition 2.5], for $\nu>0$, there exist $C, \theta \geq 1$ such that

$$
\sup _{z \in B\left(x_{B}, r\right)} h_{t}(y, z) \leq C \inf _{z \in B\left(x_{B}, r\right)} h_{\theta t}(y, z),
$$

provided $r^{m} \leq \nu t$. We take $\nu=4^{m}, r=4 r_{B}$ and $t=t_{B}=r_{B}^{m}$, to get

$$
\begin{aligned}
\sup _{z \in 4 B} h_{t_{B}}(y, z) \mu(4 B) & \leq C \int_{4 B} \inf _{z \in 4 B} h_{\theta t_{B}}(y, z) d \mu(\xi) \\
& \leq C \int_{\mathcal{X}} h_{\theta t_{B}}(y, \xi) d \mu(\xi) \leq C,
\end{aligned}
$$


independently of $\theta, t_{B}, y$. We have eventually obtained

$$
\frac{1}{\mu(B)} \int_{B}\left|D_{t_{B}}\left(f \chi_{4 B}\right)(y)\right| d \mu(y) \leq C \mathcal{M} f(x) .
$$

On the other hand, we use (5) and (6) to observe

$$
\frac{1}{\mu(B)} \int_{B}\left|D_{t_{B}}\left(f \chi_{(4 B)^{\mathrm{c}}}\right)(y)\right| d \mu(y) \leq C \frac{1}{\mu(B)} \int_{B} \mathcal{M}^{\mathrm{c}}\left(f \chi_{(4 B)^{\mathrm{c}}}\right)(y) d \mu(y) .
$$

For $y \in B$ and $0<r<3 r_{B}$ we have $B(y, r) \subset 4 B$. But, if $r \geq 3 r_{B}$ then $x \in B(y, r)$. These facts yield $\mathcal{M}^{\mathrm{c}}\left(f \chi_{(4 B) \mathrm{c}}\right)(y) \leq \mathcal{M} f(x)$ for every $y \in B$ and hence

$$
\frac{1}{\mu(B)} \int_{B}\left|D_{t_{B}}\left(f \chi_{(4 B)^{\mathrm{c}}}\right)(y)\right| d \mu(y) \leq C \mathcal{M} f(x) .
$$

We complete the proof by collecting the estimates obtained.

Corollary 3.6. If $f \in L^{p}(\mathcal{X})$ for some $1 \leq p \leq \infty$, then $\mathcal{M}_{D}^{\sharp} f(x) \leq$ $C \mathcal{M} f(x)$.

4. A good- $\lambda$ inequality. In this section we prove a good- $\lambda$ inequality for $\mathcal{M}_{D}^{\sharp}$ and $\mathcal{M}$ which allows us to obtain an analog of the classical Fefferman-Stein estimate (see $[\mathrm{FS}]$ and $[\mathrm{CF}]$ ) for our new sharp maximal function. For the genesis of the good- $\lambda$ inequalities the reader is referred to [BG]. This Fefferman-Stein type estimate for $\mathcal{M}_{D}^{\sharp}$ says that for every $\mathcal{A}_{\infty}$ weight $w$ and for every $0<p<\infty, \mathcal{M}_{D}^{\sharp}$ controls $\mathcal{M}$ in the $L^{p}(w)$ norm. In the particular case of the "approximations of the identity" considered in Proposition 3.1, the inequality that we are going to get improves the one in $[\mathrm{FS}]$ and $[\mathrm{CF}]$.

Proposition 4.1. Take $\lambda>0, f \in L_{0}^{1}(\mathcal{X})$ and a ball $B_{0}$ such that there exists $x_{0} \in B_{0}$ with $\mathcal{M} f\left(x_{0}\right) \leq \lambda$. Then, for every $0<\eta<1$, we can find $\gamma>0$ (independent of $\left.\lambda, B_{0}, f, x_{0}\right)$ in such a way that

$$
\mu\left\{x \in B_{0}: \mathcal{M} f(x)>A \lambda, \mathcal{M}_{D}^{\sharp} f(x) \leq \gamma \lambda\right\} \leq \eta \mu\left(B_{0}\right),
$$

where $A>1$ is a fixed constant which only depends on the space and the "approximation of the identity" $\left\{D_{t}: t>0\right\}$. Furthermore, for every $w \in \mathcal{A}_{\infty}$, there exist $C_{w}, r>0$ (which only depend on $w$ ) such that

$$
w\left\{x \in B_{0}: \mathcal{M} f(x)>A \lambda, \mathcal{M}_{D}^{\sharp} f(x) \leq \gamma \lambda\right\} \leq C_{w} \eta^{r} w\left(B_{0}\right) .
$$

Proof. Let us observe that the second part arises as a consequence of the first one, since it is well known (see [ST] for instance) that for every $w \in \mathcal{A}_{\infty}$, there exist $C_{w}, r>0$ such that

$$
\frac{w(E)}{w(B)} \leq C_{w}\left(\frac{\mu(E)}{\mu(B)}\right)^{r} \quad \text { for every ball } B \text { and every } E \subset B \text {. }
$$


Taking $E=\left\{x \in B_{0}: \mathcal{M} f(x)>A \lambda, \mathcal{M}_{D}^{\sharp} f(x) \leq \gamma \lambda\right\}$ and $B=B_{0}$, we can use the estimate for $\mu$ in order to obtain $w(E) \leq C_{w} \eta^{r} w\left(B_{0}\right)$.

We only work with the unweighted case. Take $\widetilde{\gamma}>0$ and

$$
E=\left\{x \in B_{0}: \mathcal{M} f(x)>A \lambda, \mathcal{M}_{D}^{\sharp} f(x) \leq \widetilde{\gamma} \lambda\right\} .
$$

Assume that we have $x_{E} \in E$ (otherwise, there is nothing to prove). By (5), for $x \in E$ we have $\widetilde{A} \lambda<\mathcal{M}^{\mathrm{c}} f(x)$, where $\widetilde{A}=\left(C_{\mu} 2^{D}\right)^{-1} A>1$ is to be chosen later. Then there exists $B\left(x, r_{x}\right)$ such that

$$
\frac{1}{\mu\left(B\left(x, r_{x}\right)\right)} \int_{B\left(x, r_{x}\right)}|f(y)| d \mu(y)>\widetilde{A} \lambda .
$$

Since $\widetilde{A}>1$, necessarily $x_{0} \notin B\left(x, r_{x}\right)$, which implies $r_{x}<2 r_{B_{0}}$ and $B\left(x, r_{x}\right) \subset 4 B_{0}$. Hence

$$
\widetilde{A} \lambda<\frac{1}{\mu\left(B\left(x, r_{x}\right)\right)} \int_{B\left(x, r_{x}\right)}|f(y)| d \mu(y) \leq \mathcal{M}\left(f \chi_{4 B_{0}}\right)(x),
$$

and $\mathcal{M}\left(f \chi_{4 B_{0}}\right)(x)>\widetilde{A} \lambda$ for every $x \in E$. Take $t_{0}=\left(16 r_{B_{0}}\right)^{m}=t_{16 B_{0}}$. We are going to show that there exist $C_{0}$ (depending on the space and $\left\{D_{t}: t>0\right\}$, but not on $\left.t_{0}\right)$ such that $\mathcal{M}\left(\left(D_{t_{0}} f\right) \chi_{4 B_{0}}\right)(x) \leq C_{0} \lambda$ for every $x \in E$. To do this we use the ideas of Lemma 3.5. As there, we observe that

$$
\left|D_{t_{0}}\left(f \chi_{16 B_{0}}\right)(y)\right| \leq C \mathcal{M} f\left(x_{0}\right) \leq C \lambda
$$

and

$$
\mathcal{M}\left(D_{t_{0}}\left(f \chi_{16 B_{0}}\right) \chi_{4 B_{0}}\right)(x) \leq C \lambda .
$$

On the other hand, if $y \in 4 B_{0}$, then

$$
\begin{aligned}
\left|D_{t_{0}}\left(f \chi_{\left(16 B_{0}\right)^{\mathrm{c}}}\right)(y)\right| & \leq C_{\mu} 2^{D} \mathcal{M}^{\mathrm{c}}\left(f \chi_{\left(16 B_{0}\right)^{\mathrm{c}}}\right)(y) \\
& \leq C_{\mu} 2^{D} \sup _{r \geq 12 r_{B_{0}}} \frac{1}{\mu(B(y, r))} \int_{B(y, r)}|f(y)| \chi_{\left(16 B_{0}\right)^{\mathrm{c}}}(y) d \mu(y) \\
& \leq C_{\mu} 2^{D} \mathcal{M} f\left(x_{0}\right) \leq C_{\mu} 2^{D} \lambda .
\end{aligned}
$$

Observe that in the first inequality we used (5) and (6); for the second we took into account that the integral vanishes for every $r<12 r_{B_{0}}$ because $B(y, r) \subset 16 B_{0}$; and the third one holds since $x_{0} \in B(y, r)$ for all $r \geq 12 r_{B_{0}}$. Then

$$
\mathcal{M}\left(D_{t_{0}}\left(f \chi_{\left(16 B_{0}\right)^{\mathrm{c}}}\right) \chi_{4 B_{0}}\right)(x) \leq C \lambda .
$$

This estimate and (10) provide $\mathcal{M}\left(\left(D_{t_{0}} f\right) \chi_{4 B_{0}}\right)(x) \leq C_{0} \lambda$, where $C_{0}$ is a constant that only depends on the space and the "approximation of the identity" $\left\{D_{t}: t>0\right\}$. Thereby, we have proved that for all $x \in E$,

$$
\mathcal{M}\left(f \chi_{4 B_{0}}\right)(x)>\widetilde{A} \lambda \text { and } \mathcal{M}\left(\left(D_{t_{0}} f\right) \chi_{4 B_{0}}\right)(x) \leq C_{0} \lambda .
$$


Choose $\widetilde{A}=C_{0}+1>1$. If $x \in E$, we have

$$
\begin{aligned}
\left(C_{0}+1\right) \lambda & <\mathcal{M}\left(f \chi_{4 B_{0}}\right)(x) \leq \mathcal{M}\left(\left(f-D_{t_{0}} f\right) \chi_{4 B_{0}}\right)(x)+\mathcal{M}\left(\left(D_{t_{0}} f\right) \chi_{4 B_{0}}\right)(x) \\
& \leq \mathcal{M}\left(\left(f-D_{t_{0}} f\right) \chi_{4 B_{0}}\right)(x)+C_{0} \lambda
\end{aligned}
$$

and so $\mathcal{M}\left(\left(f-D_{t_{0}} f\right) \chi_{4 B_{0}}\right)(x)>\lambda$. This proves that

$$
E \subset\left\{x \in B_{0}: \mathcal{M}\left(\left(f-D_{t_{0}} f\right) \chi_{4 B_{0}}\right)(x)>\lambda\right\}
$$

and by weak type $(1,1)$ inequality for the Hardy-Littlewood maximal function:

$$
\begin{aligned}
\mu(E) & \leq \frac{C}{\lambda} \int_{\mathcal{X}}\left|f(y)-D_{t_{0}} f(y)\right| \chi_{4 B_{0}}(y) d \mu(y) \leq \frac{C}{\lambda} \mu\left(16 B_{0}\right) \mathcal{M}_{D}^{\sharp} f\left(x_{E}\right) \\
& \leq C \widetilde{\gamma} \mu\left(B_{0}\right),
\end{aligned}
$$

since $t_{0}=t_{16 B_{0}}, x_{E} \in E \subset B_{0}$ and $\mathcal{M}_{D}^{\sharp} f\left(x_{E}\right) \leq \widetilde{\gamma} \lambda$. Let us point out that this estimate holds for every $\widetilde{\gamma}>0$. In this way, given $0<\eta<1$, we take $\gamma$ such that $\gamma=\eta / C$ (which is clearly independent of $\lambda, B_{0}, f, x_{0}$ ). The inequality above for this value $\gamma$ turns out to be the one we indeed want to get.

Now we can prove the analog of the classical Fefferman-Stein inequality. For the moment, we state the result just for locally integrable functions. Afterwards, we will extend it to a wider class of functions.

TheOrem 4.2. Let $0<p<\infty$ and $w \in \mathcal{A}_{\infty}$. For every $f \in L_{0}^{1}(\mathcal{X})$ with $\mathcal{M} f \in L^{p}(\mathcal{X}, w)$ it follows that:

(i) $\|\mathcal{M} f\|_{L^{p}(\mathcal{X}, w)} \leq C\left\|\mathcal{M}_{D}^{\sharp} f\right\|_{L^{p}(\mathcal{X}, w)}$ if $\mathcal{X}$ is unbounded.

(ii) $\|\mathcal{M} f\|_{L^{p}(\mathcal{X}, w)} \leq C\left\|\mathcal{M}_{D}^{\sharp} f\right\|_{L^{p}(\mathcal{X}, w)}+C\|f\|_{L^{1}(\mathcal{X})}$ if $\mathcal{X}$ is bounded.

We point out that the assumption $\mathcal{M} f \in L^{p}(\mathcal{X}, w)$ guarantees that the left-hand sides of these inequalities are finite.

Proof. Set $\widetilde{E}_{\lambda}=\{x \in \mathcal{X}: \mathcal{M} f(x)>\lambda\}$. Note that in the notation of the Calderón-Zygmund decomposition (Theorem 2.1), $\widetilde{E}_{\lambda}=E_{\lambda / C_{\mathcal{X}}}$. Fix $\tau=$ $C_{\mathcal{X}}\|f\|_{L^{1}(\mathcal{X})}(\mu(\mathcal{X}))^{-1}$ —if $\mathcal{X}$ is unbounded, then $\tau=0$ - and let us consider $\lambda>\tau$. We can perform the Calderón-Zygmund decomposition: there exists a collection $\left\{B_{i}\right\}$ of balls such that $\widetilde{E}_{\lambda}=\bigcup_{i} B_{i}$. We write $\widetilde{B}_{i}=\varepsilon_{0} B_{i}$ where $\varepsilon_{0}>1$ is the constant that appears in Remark 2.2(ii). Then, there exists $\widetilde{x}_{i} \in \widetilde{B}_{i} \backslash \widetilde{E}_{\lambda}$, that is, $\mathcal{M} f\left(\widetilde{x}_{i}\right) \leq \lambda$. Let us use Proposition 4.1: there are $C_{w}, r>0$ (which only depend on $w$ ) and $A>1$ (which depends on $\mathcal{X}$, $\left\{D_{t}: t>0\right\}$ ) such that, if $0<\eta<1$ (to be chosen later), we can find $\gamma>0$ (independent of $\lambda, f, \widetilde{B}_{i}$ ) in such a way that

$$
w\left\{x \in \widetilde{B}_{i}: \mathcal{M} f(x)>A \lambda, \mathcal{M}_{D}^{\sharp} f(x) \leq \gamma \lambda\right\} \leq C_{w} \eta^{r} w\left(\widetilde{B}_{i}\right) .
$$


Set $U_{\lambda}=\left\{x \in \mathcal{X}: \mathcal{M} f(x)>A \lambda, \mathcal{M}_{D}^{\sharp} f(x) \leq \gamma \lambda\right\}$ and so $U_{\lambda} \subset \widetilde{E}_{\lambda}=$ $\bigcup_{i} B_{i} \subset \bigcup_{i} \widetilde{B}_{i}$ since $A>1$. Then, for any $\lambda>\tau$,

$$
\begin{aligned}
w\left(U_{\lambda}\right) & \leq \sum_{i} w\left\{x \in \widetilde{B}_{i}: \mathcal{M} f(x)>A \lambda, \mathcal{M}_{D}^{\sharp} f(x) \leq \gamma \lambda\right\} \\
& \leq C_{w} \eta^{r} \sum_{i} w\left(\widetilde{B}_{i}\right) \\
& \leq C_{w} \eta^{r} C_{w, \varepsilon_{0}} \sum_{i} w\left(B_{i}\right) \leq C \eta^{r} M w\left(\bigcup_{i} B_{i}\right)=C \eta^{r} w\left(\widetilde{E}_{\lambda}\right) \\
& =C \eta^{r} w\{x \in \mathcal{X}: \mathcal{M} f(x)>\lambda\},
\end{aligned}
$$

where we used the fact that $\mathcal{A}_{\infty}$ weights are doubling measures, $M$ is the constant in (iv) of Theorem 2.1, and $C$ is a constant that only depends on the space and the weight.

We first handle the case where $\mathcal{X}$ is unbounded. Then $\tau=0$ and the previous inequality holds for every $\lambda>0$. Because of the fact that $0<p$ $<\infty$, and by (11), one can prove that

$$
\begin{aligned}
\|\mathcal{M} f\|_{L^{p}(\mathcal{X}, w)}^{p} & =A^{p} \int_{0}^{\infty} p \lambda^{p-1} w\{x \in \mathcal{X}: \mathcal{M} f(x)>A \lambda\} d \lambda \\
& \leq A^{p} \int_{0}^{\infty} p \lambda^{p-1}\left(w\left(U_{\lambda}\right)+w\left\{x \in \mathcal{X}: \mathcal{M}_{D}^{\sharp} f(x)>\gamma \lambda\right\}\right) d \lambda \\
& \leq(C+1) A^{p} \eta^{r}\|\mathcal{M} f\|_{L^{p}(\mathcal{X}, w)}^{p}+\frac{A^{p}}{\gamma^{p}}\left\|\mathcal{M}_{D}^{\sharp} f\right\|_{L^{p}(\mathcal{X}, w)}^{p} .
\end{aligned}
$$

Observe that both terms are finite since by hypothesis $\mathcal{M} f \in L^{p}(\mathcal{X}, w)$ and by Corollary 3.6, $\mathcal{M}_{D}^{\sharp} f$ also belongs to this space. Let us choose $\eta$ such that $(C+1) A^{p} \eta^{r}=1 / 2$ (it is clear that $0<\eta<1$ and that $\eta$ only depends on $\mathcal{X}$, $w$ and $\left.\left\{D_{t}: t>0\right\}\right)$. The former inequality turns out to be

$$
\|\mathcal{M} f\|_{L^{p}(\mathcal{X}, w)}^{p} \leq 2 \frac{A^{p}}{\gamma^{p}}\left\|\mathcal{M}_{D}^{\sharp} f\right\|_{L^{p}(\mathcal{X}, w)}^{p} .
$$

When $\mathcal{X}$ is bounded we have $\mathcal{X}=B\left(x_{0}, r_{0}\right)$ and, since $w \in L_{\text {loc }}^{1}(\mathcal{X})$ (because $\left.w \in \mathcal{A}_{\infty}\right)$ it follows that $w(\mathcal{X})<\infty$. Now we can only use CalderónZygmund decomposition and (11) for $\lambda>\tau$. Thereby,

$$
\begin{aligned}
\|\mathcal{M} f\|_{L^{p}(\mathcal{X}, w)}^{p} & =A^{p} \int_{0}^{\infty} p \lambda^{p-1} w\{x \in \mathcal{X}: \mathcal{M} f(x)>A \lambda\} d \lambda \\
= & A^{p}\left(\int_{0}^{\tau} \ldots d \lambda+\int_{\tau}^{\infty} \ldots d \lambda\right) \\
\leq & A^{p} \tau^{p} w(\mathcal{X})+A^{p}(C+1) \eta^{r}\|\mathcal{M} f\|_{L^{p}(\mathcal{X}, w)}^{p}+\frac{A^{p}}{\gamma^{p}}\left\|\mathcal{M}_{D}^{\sharp} f\right\|_{L^{p}(\mathcal{X}, w)}^{p},
\end{aligned}
$$


where the estimate for the first term is trivial and for the second one we employ (11) as in the former case. Use the fact that each term is finite, take $\eta$ such that $A^{p}(C+1) \eta^{r}=1 / 2$, and plug the value of $\tau$ into the inequality above to get

$$
\|\mathcal{M} f\|_{L^{p}(\mathcal{X}, w)} \leq C\left\|\mathcal{M}_{D}^{\sharp} f\right\|_{L^{p}(\mathcal{X}, w)}+C \tau=C\left\|\mathcal{M}_{D}^{\sharp} f\right\|_{L^{p}(\mathcal{X}, w)}+C\|f\|_{L^{1}(\mathcal{X})} .
$$

Now we can extend the previous result to a wider class of functions. The only interesting case is when $\mathcal{X}$ is unbounded because otherwise the set of functions that belong to some $L^{q}(\mathcal{X})$ is just $L^{1}(\mathcal{X})=L_{0}^{1}(\mathcal{X})$.

Corollary 4.3. Assume that $\mathcal{X}$ is unbounded. Let $0<p<\infty, w \in \mathcal{A}_{\infty}$ and $f \in L^{q}(\mathcal{X})$ for some $1 \leq q \leq \infty$. If $\mathcal{M} f \in L^{p}(\mathcal{X}, w)$, then

$$
\int_{\mathcal{X}}(\mathcal{M} f(x))^{p} w(x) d \mu(x) \leq C \int_{\mathcal{X}}\left(\mathcal{M}_{D}^{\sharp} f(x)\right)^{p} w(x) d \mu(x) .
$$

Proof. Take such a function $f$. By Corollary 3.6, $\mathcal{M}_{D}^{\sharp} f(x) \leq C \mathcal{M} f(x) \in$ $L^{p}(\mathcal{X}, w)$ and the right-hand side of the inequality we want to prove is finite as well. Since $\mathcal{X}$ is unbounded $\mu(\mathcal{X})=\infty$ and standard computations show that $w(\mathcal{X})=\infty$ for $w \in \mathcal{A}_{\infty}$. Let us take $x_{0} \in \mathcal{X}$ and we are going to prove that for all $x \in \mathcal{X}, \mathcal{M}\left(f \chi_{B\left(x_{0}, R\right)^{\mathrm{c}}}\right)(x) \rightarrow 0$ as $R \rightarrow \infty$. Let us observe that $\mathcal{M}\left(f \chi_{B\left(x_{0}, R\right)^{\mathrm{c}}}\right)(x)$ decreases as $R$ increases because $\chi_{B\left(x_{0}, R\right)^{\mathrm{c}}}$ does and the previous limit always exists. Assume that we find $x_{1} \in \mathcal{X}$ such that this limit is $C_{0}>0$. In particular, $\mathcal{M}\left(f \chi_{B\left(x_{0}, R\right)^{\mathrm{c}}}\right)\left(x_{1}\right) \geq C_{0}$ for every $R>0$. For $x \in \mathcal{X}$, we define $R(x)=\max \left\{d\left(x_{1}, x_{0}\right), d\left(x, x_{0}\right)\right\}+1$ and so $x, x_{1} \in B\left(x_{0}, R(x)\right)$. If $R \geq 4 R(x)$,

$$
\begin{aligned}
& \mathcal{M}\left(f \chi_{B\left(x_{0}, R\right)^{\mathrm{c}}}\right)\left(x_{1}\right) \\
& \leq C_{\mu} 2^{D} \sup _{r \geq 3 R(x)} \frac{1}{\mu\left(B\left(x_{1}, r\right)\right)} \int_{B\left(x_{1}, r\right)}|f(y)| \chi_{B\left(x_{0}, R\right)^{\mathrm{c}}}(y) d \mu(y) \\
& \leq C_{\mu} 2^{D} \mathcal{M}\left(f \chi_{B\left(x_{0}, R\right)^{\mathrm{c}}}\right)(x),
\end{aligned}
$$

because if $r<3 R(x)$, then $B\left(x_{1}, r\right) \subset B\left(x_{0}, R\right)$ and the integral vanishes. On the other hand, the last inequality holds because $x \in B\left(x_{0}, R(x)\right) \subset$ $B\left(x_{1}, r\right)$. Then, for $R \geq 4 R(x)$, we have

$$
C_{0} \leq \mathcal{M}\left(f \chi_{B\left(x_{0}, R\right)^{\mathrm{c}}}\right)\left(x_{1}\right) \leq C_{\mu} 2^{D} \mathcal{M}\left(f \chi_{B\left(x_{0}, R\right)^{\mathrm{c}}}\right)(x) \leq C_{\mu} 2^{D} \mathcal{M} f(x),
$$

and so

$$
\int_{\mathcal{X}}(\mathcal{M} f(x))^{p} w(x) d \mu(x) \geq\left(\frac{C_{0}}{C_{\mu} 2^{D}}\right)^{p} w(\mathcal{X})=\infty,
$$

which contradicts $\mathcal{M} f \in L^{p}(\mathcal{X}, w)$. So, for all $x \in \mathcal{X}, \mathcal{M}\left(f \chi_{B\left(x_{0}, R\right)^{\mathrm{c}}}\right)(x) \rightarrow 0$ as $R \rightarrow \infty$. On the other hand, since $\mathcal{M} f \in L^{p}(\mathcal{X}, w)$, the dominated convergence theorem shows that that $\left\|\mathcal{M}\left(f \chi_{B\left(x_{0}, R_{0}\right)^{\mathrm{c}}}\right)\right\|_{L^{p}(\mathcal{X}, w)} \rightarrow 0$ as $R \rightarrow \infty$. 
Then, for any $\varepsilon>0$, there exists $R_{0}>0$ such that $\left\|\mathcal{M}\left(f \chi_{B\left(x_{0}, R_{0}\right)^{\mathrm{c}}}\right)\right\|_{L^{p}(\mathcal{X}, w)}$ $<\varepsilon$. In this way,

$$
\|\mathcal{M} f\|_{L^{p}(\mathcal{X}, w)}<\varepsilon+\left\|\mathcal{M}\left(f \chi_{B\left(x_{0}, R_{0}\right)}\right)\right\|_{L^{p}(\mathcal{X}, w)} .
$$

Since $f \in L^{q}(\mathcal{X}), q \geq 1$, it follows that $f \chi_{B\left(x_{0}, R_{0}\right)} \in L_{0}^{1}(\mathcal{X})$. On the other hand, $\mathcal{M}\left(f \chi_{B\left(x_{0}, R_{0}\right)}\right) \in L^{p}(\mathcal{X}, w)$, because $\mathcal{M} f$ belongs to the same space. We can now use Theorem 4.2 to prove that

$$
\begin{aligned}
\left\|\mathcal{M}\left(f \chi_{B\left(x_{0}, R_{0}\right)}\right)\right\|_{L^{p}(\mathcal{X}, w)} & \leq C\left\|\mathcal{M}_{D}^{\sharp}\left(f \chi_{B\left(x_{0}, R_{0}\right)}\right)\right\|_{L^{p}(\mathcal{X}, w)} \\
& \leq C\left\|\mathcal{M}_{D}^{\sharp} f\right\|_{L^{p}(\mathcal{X}, w)}+C\left\|\mathcal{M}_{D}^{\sharp}\left(f \chi_{B\left(x_{0}, R_{0}\right)^{\mathrm{c}}}\right)\right\|_{L^{p}(\mathcal{X}, w)} .
\end{aligned}
$$

Because of the fact that $f \chi_{B\left(x_{0}, R_{0}\right)^{c}} \in L^{q}(\mathcal{X})$, Corollary 3.6 leads to

$$
\left\|\mathcal{M}_{D}^{\sharp}\left(f \chi_{B\left(x_{0}, R_{0}\right)^{\mathrm{c}}}\right)\right\|_{L^{p}(\mathcal{X}, w)} \leq C\left\|\mathcal{M}\left(f \chi_{B\left(x_{0}, R\right)^{\mathrm{c}}}\right)\right\|_{L^{p}(\mathcal{X}, w)} \leq C \varepsilon .
$$

By collecting all these estimates and by using the fact that $\varepsilon$ can be taken arbitrarily small we get the desired estimate.

5. Weighted norm inequalities for singular integral operators with non-smooth kernels. The operators we are going to consider henceforth were introduced in $[\mathrm{DM}]$. They are defined in the following way:

(a) $T$ is a bounded linear operator on $L^{2}(\mathcal{X})$ with kernel $K(x, y)$ such that for $f \in L_{0}^{\infty}(\mathcal{X})$,

$$
T f(x)=\int_{\mathcal{X}} K(x, y) f(y) d \mu(y) \quad \text { for } \mu \text {-almost every } x \notin \operatorname{supp} f .
$$

(b) There exists an "approximation of the identity" $\left\{A_{t}: t>0\right\}$ such that $T A_{t}$ has associated kernel $k_{t}(x, y)$ and there exist $c_{1}, c_{2}>0$ so that

$$
\int_{d(x, y) \geq c_{1} t^{1 / m}}\left|K(x, y)-k_{t}(x, y)\right| d \mu(x) \leq c_{2} \quad \text { for all } y \in \mathcal{X} .
$$

(c) There exists an "approximation of the identity" $\left\{D_{t}: t>0\right\}$ such that $D_{t} T$ has kernel $K_{t}(x, y)$ which satisfies

$$
\begin{aligned}
& \text { (c1) }\left|K_{t}(x, y)\right| \leq c_{4} \frac{1}{\mu\left(B\left(x, t^{1 / m}\right)\right)} \text { when } d(x, y) \leq c_{3} t^{1 / m}, \\
& \text { (c2) }\left|K_{t}(x, y)-K(x, y)\right| \leq c_{4} \frac{1}{\mu(B(x, d(x, y)))} \frac{t^{\alpha / m}}{d(x, y)^{\alpha}} \\
& \text { when } d(x, y) \geq c_{3} t^{1 / m},
\end{aligned}
$$

for some $c_{3}, c_{4}, \alpha>0$ (in fact, without loss of generality in what follows we will assume that $c_{3}=1$ ). 
We assume that $T$ is an operator satisfying (a), (b) and (c). The maximal operator $T_{\star}$ is the supremum of the truncated integrals, namely,

$$
T_{\star} f(x)=\sup _{\varepsilon>0}\left|T_{\varepsilon} f(x)\right|=\sup _{\varepsilon>0}\left|\int_{d(x, y) \geq \varepsilon} K(x, y) f(y) d \mu(y)\right| .
$$

REMARK 5.1. In $[\mathrm{DM}]$, it is proved that if $T$ satisfies (a) and (b), then it is of weak type $(1,1)$ and of strong type $(p, p)$ for $1<p \leq 2$. In addition, if (c) is also assumed, then $T$ is bounded on $L^{p}(\mathcal{X}), 1<p<\infty$. Furthermore, [DM, Theorem 3] says that $T_{\star}$ is a bounded operator on $L^{p}(\mathcal{X})$, $1<p<\infty$. Indeed, implicitly in the proof we can find the following Cotlar type inequality:

$$
T_{\star} f(x) \leq C \mathcal{M}(T f)(x)+C \mathcal{M} f(x) .
$$

Then the boundedness of $T$ and $\mathcal{M}$ provides the corresponding estimates for $T_{\star}$.

REMARK 5.2. In comparison with the classical Calderón-Zygmund operators, the Hörmander conditions are replaced by (b) and (c) which involve the "approximations of the identity". Let us note that there is no regularity assumption on the space variables. In fact, Duong and McIntosh prove that, for suitable "approximations of the identity", conditions (b) and (c) are weaker than the usual imposed to Calderón-Zygmund operators. The reader is referred to $[\mathrm{DM}]$ for more details.

5.1. Weighted strong type inequalities. The goal of this section is to prove that the operators we are working with satisfy weighted strong type inequalities. The main tool will be the Fefferman-Stein type inequality for $\mathcal{M}_{D}^{\sharp}$ obtained before.

TheOREM 5.3. Let $1<p<\infty$ and $w \in \mathcal{A}_{p}$. If $T$ is an operator satisfying (a), (b) and (c), then $T$ is bounded on $L^{p}(\mathcal{X}, w)$.

For the proof of this result, most of the work is already done. We are going to show that $\mathcal{M}_{D}^{\sharp}(T f)$ is pointwise controlled by the Hardy-Littlewood maximal function with some power strictly bigger than 1 . Let us point out that $\mathcal{M}_{D}^{\sharp}$ is the ad hoc operator for this property and it plays the same role as the Fefferman-Stein sharp maximal function with respect to Calderón-Zygmund operators. Then Theorem 4.2, Corollary 4.3, and a technical lemma will allow us to obtain the weighted norm estimates.

Proposition 5.4. Let $1<s<\infty$ and $f \in L_{0}^{\infty}(\mathcal{X})$. If $T$ is an operator as above then

$$
\mathcal{M}_{D}^{\sharp}(T f)(x) \leq C\left(\mathcal{M}\left(|f|^{s}\right)(x)\right)^{1 / s} .
$$


Proof. Let us note that, if $f \in L_{0}^{\infty}(\mathcal{X})$, then $f \in L^{p}(\mathcal{X})$ for every $1 \leq$ $p \leq \infty$ and $\mathcal{M}\left(|f|^{s}\right)$ makes sense. Moreover, because of the boundedness of $T$ obtained in [DM] (see Remark 5.1), it follows that $T f \in L^{p}(\mathcal{X})$ for $1<p<\infty$ and $\mathcal{M}_{D}^{\sharp}(T f)$ also makes sense. For every ball $B \ni x$, we split $f$ as $f=f_{1}+f_{2}=f \chi_{2 B}+f \chi_{(2 B)^{c}}$. Since $1<s<\infty, T$ is bounded on $L^{s}(\mathcal{X})$ and

$$
\frac{1}{\mu(B)} \int_{B}\left|T f_{1}(y)\right| d \mu(y) \leq C\left(\frac{1}{\mu(B)} \int_{\mathcal{X}}\left|f_{1}(y)\right|^{s} d \mu(y)\right)^{1 / s} \leq C\left(\mathcal{M}\left(|f|^{s}\right)(x)\right)^{1 / s}
$$

because $x \in B \subset 2 B$. On the other hand, (6) and the boundedness of $\mathcal{M}$ on $L^{s}(\mathcal{X})$ provide in the same manner

$$
\begin{aligned}
\frac{1}{\mu(B)} \int_{B}\left|D_{t_{B}}\left(T f_{1}\right)(y)\right| d \mu(y) & \leq C \frac{1}{\mu(B)} \int_{B}\left|\mathcal{M}\left(T f_{1}\right)(y)\right| d \mu(y) \\
& \leq C\left(\mathcal{M}\left(|f|^{s}\right)(x)\right)^{1 / s} .
\end{aligned}
$$

Let us see what happens with $f_{2}$. We know that this function is boundedly supported, because $f \in L_{0}^{\infty}(\mathcal{X})$ is, and the support is contained in $(2 B)^{\mathrm{c}}$. Let $y \in B$. If $z \in(2 B)^{\mathrm{c}}$, then $d(z, y) \geq r_{B}=t_{B}^{1 / m}$. By (c2), we obtain

$$
\begin{aligned}
\mid T f_{2}(y) & -D_{t_{B}}\left(T f_{2}\right)(y) \mid \\
& \leq \int_{d(z, y) \geq t_{B}^{1 / m}}\left|K(y, z)-K_{t_{B}}(y, z)\right||f(z)| d \mu(z) \\
& \leq C \sum_{k=0}^{\infty} \int_{2^{k} r_{B} \leq d(z, y)<2^{k+1} r_{B}} \frac{1}{\mu(B(y, d(y, z)))} \frac{r_{B}^{\alpha}}{d(y, z)^{\alpha}}|f(z)| d \mu(z) \\
& \leq C \sum_{k=0}^{\infty} \frac{1}{2^{k \alpha}} \frac{1}{\mu\left(B\left(y, 2^{k} r_{B}\right)\right)} \int_{d(z, y)<2^{k+1} r_{B}}|f(z)| d \mu(z) \\
& \leq C \mathcal{M} f(x),
\end{aligned}
$$

since $x \in B \subset B\left(y, 2^{k+1} r_{B}\right)$. Then, as $s>1$,

$$
\frac{1}{\mu(B)} \int_{B}\left|T f_{2}(y)-D_{t_{B}}\left(T f_{2}\right)(y)\right| d \mu(y) \leq C \mathcal{M} f(x) \leq C\left(\mathcal{M}\left(|f|^{s}\right)(x)\right)^{1 / s} .
$$

To complete the proof we just need to paste these three estimates.

Before proving the weighted norm inequalities we need a technical lemma to be proved later. We see, for good functions $f$, that $T f$ belongs to $L^{p}(\mathcal{X}, w)$. That is, we obtain an a priori estimate which says that the left-hand side of the weighted inequality is finite. This fact will allow us to use Theorem 4.2 and Corollary 4.3. 
Lemma 5.5. Let $1<p<\infty, w \in \mathcal{A}_{p}$ and $f \in L_{0}^{\infty}(\mathcal{X})$. Then $T f \in$ $L^{p}(\mathcal{X}, w)$.

Proof of Theorem 5.3. It is enough to prove the desired estimate for $f \in L_{0}^{\infty}(\mathcal{X})$, since this space is densely contained in $L^{p}(\mathcal{X}, w)$. For $w \in \mathcal{A}_{p}$, there exists $s>1$ such that $w \in \mathcal{A}_{p / s}$ with $p / s>1$. On the other hand, $f \in L^{q}(\mathcal{X})$ for $q \geq 1$ and the estimates on $T$ ensure that $T f \in L^{q}(\mathcal{X})$ for $1<q<\infty$. By Lebesgue's differentiation theorem $T f(x) \leq \mathcal{M}(T f)(x)$ for $\mu$-almost every $x \in \mathcal{X}$.

Let us consider first the case where $\mathcal{X}$ is unbounded. Lemma 5.5 says that $T f \in L^{p}(\mathcal{X}, w)$. Since $w \in \mathcal{A}_{p}$, we see that $\mathcal{M}$ is bounded on $L^{p}(\mathcal{X}, w)$ and consequently $\mathcal{M}(T f)$ also belongs to this space. We use these facts, Corollary 4.3 and Proposition 5.4 with our choice of $s$ to obtain

$$
\begin{aligned}
\int_{\mathcal{X}}|T f(x)|^{p} w(x) d \mu(x) & \leq \int_{\mathcal{X}}(\mathcal{M}(T f)(x))^{p} w(x) d \mu(x) \\
& \leq C \int_{\mathcal{X}}\left(\mathcal{M}_{D}^{\sharp}(T f)(x)\right)^{p} w(x) d \mu(x) \\
& \leq C \int_{\mathcal{X}}\left(\mathcal{M}\left(|f|^{s}\right)(x)\right)^{p / s} w(x) d \mu(x) \\
& \leq C \int_{\mathcal{X}}|f(x)|^{p} w(x) d \mu(x) .
\end{aligned}
$$

In the last inequality we used the fact that $\mathcal{M}$ is bounded on $L^{p / s}(\mathcal{X}, w)$ since $w \in \mathcal{A}_{p / s}$ and $p / s>1$.

When $\mathcal{X}$ is a bounded set we proceed as follows. Since $T f \in L^{q}(\mathcal{X})$ for $q>1$ and $\mu(\mathcal{X})<\infty$, it follows that $T f \in L^{1}(\mathcal{X})$. Moreover, since the space is bounded, in particular the support of $T f$ is also a bounded set. Apply Lemma 5.5 to get $T f \in L^{p}(\mathcal{X}, w)$, and hence $\mathcal{M}(T f)$ belongs to this space. As before, we can use Theorem 4.2 and Proposition 5.4 to obtain

$$
\begin{aligned}
\|T f\|_{L^{p}(\mathcal{X}, w)} & \leq\|\mathcal{M}(T f)\|_{L^{p}(\mathcal{X}, w)} \leq C\left\|\mathcal{M}_{D}^{\sharp}(T f)\right\|_{L^{p}(\mathcal{X}, w)}+C\|T f\|_{L^{1}(\mathcal{X})} \\
& \leq C\|f\|_{L^{p}(\mathcal{X}, w)}+C\|T f\|_{L^{1}(\mathcal{X})} .
\end{aligned}
$$

For the second term, Hölder's inequality with $s>1$ and the boundedness of $T$ in $L^{s}(\mathcal{X})$ yield $\|T f\|_{L^{1}(\mathcal{X})} \leq \mu(\mathcal{X})^{1 / s^{\prime}}\|T f\|_{L^{s}(\mathcal{X})} \leq C\|f\|_{L^{s}(\mathcal{X})}$. We again apply Hölder's inequality with exponent $r=p / s>1$ to observe that

$$
\|f\|_{L^{s}(\mathcal{X})} \leq\left(\int_{\mathcal{X}}|f(x)|^{p} w(x) d \mu(x)\right)^{1 / p}\left(\int_{\mathcal{X}} w(x)^{1-r^{\prime}} d \mu(x)\right)^{(r-1) / p} .
$$

As $w \in \mathcal{A}_{p / s}=\mathcal{A}_{r}$, we know that $w^{1-r^{\prime}} \in \mathcal{A}_{r^{\prime}}$. In particular $w^{1-r^{\prime}} \in$ $L_{\text {loc }}^{1}(\mathcal{X})$ and then the second factor of the previous inequality is finite (let us 
recall that $\mathcal{X}$ is bounded). To finish, it is enough to collect all the estimates obtained.

For the proof of Lemma 5.5 we will use the following:

Remark 5.6. Let $1<q<\infty$ and $w \in \mathcal{A}_{q}$. Then, for any $x_{0} \in \mathcal{X}$ and $R>0$, we have

$$
\sum_{k=0}^{\infty} \int_{2^{k} R \leq d\left(x, x_{0}\right)<2^{k+1} R} \frac{1}{\mu\left(B\left(x, 2^{k} R\right)\right)^{q}} w(x) d \mu(x)<\infty .
$$

We prove this fact in a very easy way. Take $g=\chi_{B\left(x_{0}, R\right)}$. Since $w \in \mathcal{A}_{q}$,

$$
\int_{\mathcal{X}}(\mathcal{M} g(x))^{q} w(x) d \mu(x) \leq C \int_{\mathcal{X}}|g(x)|^{q} w(x) d \mu(x)=C w\left(B\left(x_{0}, R\right)\right)<\infty .
$$

For $k=0,1, \ldots$ and $x \in \mathcal{X}$ such that $2^{k} R \leq d\left(x, x_{0}\right)<2^{k+1} R$, we observe that $B\left(x_{0}, R\right) \subset B\left(x, 2^{k+2} R\right)$ and thus

$$
\mathcal{M} g(x) \geq \frac{1}{\mu\left(B\left(x, 2^{k+2} R\right)\right)} \int_{B\left(x, 2^{k+2} R\right)}|g(y)| d \mu(y)=\frac{\mu\left(B\left(x_{0}, R\right)\right)}{\mu\left(B\left(x, 2^{k} R\right)\right)} .
$$

Finally,

$$
\begin{aligned}
\infty & >\int_{\mathcal{X}}(\mathcal{M} g(x))^{q} w(x) d \mu(x) \geq \sum_{k=0}^{\infty} \int_{2^{k} R \leq d\left(x, x_{0}\right)<2^{k+1} R}(\mathcal{M} g(x))^{q} w(x) d \mu(x) \\
& \geq C \sum_{k=0}^{\infty} \int_{2^{k} R \leq d\left(x, x_{0}\right)<2^{k+1} R} \frac{1}{\mu\left(B\left(x, 2^{k} R\right)\right)^{q}} w(x) d \mu(x) .
\end{aligned}
$$

Proof of Lemma 5.5. We know that $\operatorname{supp} f \subset B\left(x_{0}, R\right)$ for some $x_{0} \in \mathcal{X}$ and $R>0$. Reverse Hölder's inequality (see [ST]) provides $\varepsilon>0$ such that

$$
\left(\frac{1}{\mu(B)} \int_{B} w(x)^{1+\varepsilon} d \mu(x)\right)^{1 /(1+\varepsilon)} \leq \frac{C}{\mu(B)} \int_{B} w(x) d \mu(x),
$$

holds for every ball $B$. By Hölder's inequality with $1+\varepsilon$, we obtain

$$
\begin{aligned}
\int_{B\left(x_{0}, 2 R\right)}|T f(x)|^{p} w(x) d \mu(x) \\
\leq\left(\int_{B\left(x_{0}, 2 R\right)} w(x)^{1+\varepsilon} d \mu(x)\right)^{1 /(1+\varepsilon)}\|T f\|_{L^{p(1+\varepsilon)^{\prime}(\mathcal{X})}}^{p} \\
\leq C \frac{w\left(B\left(x_{0}, 2 R\right)\right)}{\mu\left(B\left(x_{0}, 2 R\right)\right)^{\varepsilon /(1+\varepsilon)}}\|f\|_{L^{p(1+\varepsilon)^{\prime}(\mathcal{X})}}^{p}<\infty
\end{aligned}
$$


because $f \in L_{0}^{\infty}(\mathcal{X})$ and $T$ is bounded on $L^{p(1+\varepsilon)^{\prime}}(\mathcal{X})$ (see $[\mathrm{DM}]$ or Remark 5.1). Take $t_{k}=\left(2^{k-1} R\right)^{m}$ and it follows that

$$
\begin{aligned}
\int_{B\left(x_{0}, 2 R\right)^{\mathrm{c}}} \mid & \left.T f(x)\right|^{p} w(x) d \mu(x) \\
= & \sum_{k=1}^{\infty} \int_{2^{k} R \leq d\left(x, x_{0}\right)<2^{k+1} R}|T f(x)|^{p} w(x) d \mu(x) \\
\leq & C_{p}\left(\sum_{k=1}^{\infty} \int_{2^{k}} \int_{R \leq d\left(x, x_{0}\right)<2^{k+1} R}\left|T f(x)-D_{t_{k}} T f(x)\right|^{p} w(x) d \mu(x)\right. \\
& \left.+\sum_{k=1}^{\infty} \int_{2^{k} R \leq d\left(x, x_{0}\right)<2^{k+1} R}\left|D_{t_{k}} T f(x)\right|^{p} w(x) d \mu(x)\right) \\
= & C_{p}(I+I I) .
\end{aligned}
$$

For the first term, if $2^{k} R \leq d\left(x, x_{0}\right)<2^{k+1} R$ and $y \in B\left(x_{0}, R\right)$, we have $d(x, y) \geq t_{k}^{1 / m}$. Then $(\mathrm{c} 2)$ provides

$$
\begin{aligned}
& \left|T f(x)-D_{t_{k}} T f(x)\right| \\
& \quad \leq C \int_{d(x, y) \geq t_{k}^{1 / m}} \frac{1}{\mu(B(x, d(x, y)))} \frac{t_{k}^{\alpha / m}}{d(x, y)^{\alpha}}|f(y)| d \mu(y) \\
& \quad \leq C \sum_{j=0}^{\infty} \frac{1}{2^{j \alpha}} \frac{1}{\mu\left(B\left(x, 2^{j+1} t_{k}^{1 / m}\right)\right)} \int_{2^{j} t_{k}^{1 / m} \leq d(x, y)<2^{j+1} t_{k}^{1 / m}}|f(y)| d \mu(y) \\
& \quad \leq C \mathcal{M} f(x),
\end{aligned}
$$

since $\alpha>0$. As $w \in \mathcal{A}_{p}$, we have

$$
\begin{aligned}
I & \leq C \sum_{k=1}^{\infty} \int_{2^{k}} \int_{R \leq d\left(x, x_{0}\right)<2^{k+1} R}(\mathcal{M} f(x))^{p} w(x) d \mu(x) \\
& \leq C \int_{\mathcal{X}}(\mathcal{M} f(x))^{p} w(x) d \mu(x) \\
& \leq C \int_{\mathcal{X}}|f(x)|^{p} w(x) d \mu(x) \leq C\|f\|_{L^{\infty}(\mathcal{X})}^{p} w\left(B\left(x_{0}, R\right)\right)<\infty .
\end{aligned}
$$

Let us estimate $I I$. First, we use again the openness property of the Muckenhoupt classes, and the fact that there exists $1<s<\infty$ such that $w \in \mathcal{A}_{p / s}$ with $p / s>1$. Since $f \in L_{0}^{\infty}(\mathcal{X})$, we have $f \in L^{s}(\mathcal{X})$ and consequently $T f \in L^{s}(\mathcal{X})$. We use Lemma 3.4 to obtain 


$$
\begin{aligned}
I I & \leq C\|T f\|_{L^{s}(\mathcal{X})}^{p} \sum_{k=1}^{\infty} \int_{2^{k}}^{\infty \leq d\left(x, x_{0}\right)<2^{k+1} R} \frac{1}{\mu\left(B\left(x, t_{k}^{1 / m}\right)\right)^{p / s}} w(x) d \mu(x) \\
& \leq C\|f\|_{L^{s}(\mathcal{X})}^{p} \sum_{k=1}^{\infty} \int_{2^{k}{ }_{R \leq d\left(x, x_{0}\right)<2^{k+1} R}} \frac{1}{\mu\left(B\left(x, 2^{k} R\right)\right)^{p / s}} w(x) d \mu(x)<\infty
\end{aligned}
$$

because of Remark 5.6 with $q=p / s$. Thus we have shown that outside of $B\left(x_{0}, 2 R\right)$ the integral is also finite.

COROLlary 5.7. If $T$ is an operator satisfying (a), (b), and (c) then $T_{\star}$ is bounded on $L^{p}(\mathcal{X}, w)$ for $1<p<\infty$ and $w \in \mathcal{A}_{p}$.

Proof. Use the Cotlar type inequality (12) proved in [DM], Theorem 5.3, and the boundedness of $\mathcal{M}$ on $L^{p}(\mathcal{X}, w)$ for $w \in \mathcal{A}_{p}, 1<p<\infty$.

5.2. Weighted weak type $(1,1)$ inequality. By means of the CalderónZygmund decomposition and the strong type estimates we have just proved, we can now obtain the corresponding weak type $(1,1)$ inequality for $\mathcal{A}_{1}$ weights.

THEOREM 5.8. Let $T$ be an operator satisfying (a) and (c). Assume that, instead of (b), T satisfies the following stronger condition:

$(\mathrm{b})^{\prime}$ There exists an "approximation of the identity" $\left\{A_{t}: t>0\right\}$ such that $T A_{t}$ has associated kernel $k_{t}(x, y)$ and there are some $c_{1}, c_{2}, \beta>0$ such that

$$
\left|K(x, y)-k_{t}(x, y)\right| \leq c_{2} \frac{1}{\mu(B(y, d(x, y)))} \frac{t^{\beta / m}}{d(x, y)^{\beta}}
$$

for $x, y \in \mathcal{X}$ with $d(x, y) \geq c_{1} t^{1 / m}$.

Then, for every $w \in \mathcal{A}_{1}, T$ maps $L^{1}(\mathcal{X}, w)$ into $L^{1, \infty}(\mathcal{X}, w)$.

REMARK 5.9. Hypothesis (b)' implies that if $g \in L_{\text {loc }}^{1}(\mathcal{X})$, then

$$
\int_{d(x, y) \geq c_{1} t^{1 / m}}\left|K(x, y)-k_{t}(x, y)\right||g(x)| d \mu(x) \leq C \mathcal{M} g(y) \quad \text { for any } y \in \mathcal{X} .
$$

In particular, by taking $g(x)=1$, it follows that (b)' implies (b).

The idea consists in decomposing the integral into dyadic annuli:

$$
\begin{aligned}
& \quad \int_{d(x, y) \geq c_{1} t^{1 / m}}\left|K(x, y)-k_{t}(x, y)\right||g(x)| d \mu(x) \\
& \leq C \sum_{k=0}^{\infty} \frac{1}{2^{k \beta}} \frac{1}{\mu\left(B\left(y, 2^{k} c_{1} t^{1 / m}\right)\right)} \int_{2^{k} c_{1} t^{1 / m} \leq d(x, y)<2^{k+1} c_{1} t^{1 / m}}|g(x)| d \mu(x) \\
& \leq C \mathcal{M} g(y) .
\end{aligned}
$$


Proof of Theorem 5.8. We know that $w \in \mathcal{A}_{1} \subset \mathcal{A}_{p}$ for every $1<p<\infty$ and, by the remark above, $T$ satisfies the hypotheses of Theorem 5.3. Then $T$ is bounded on $L^{p}(\mathcal{X}, w)$. On the other hand, it is enough to prove the desired inequality for $f \in L_{0}^{\infty}(\mathcal{X})$. If $\lambda>\|f\|_{L^{1}(\mathcal{X})}(\mu(\mathcal{X}))^{-1}$, by Theorem 2.1, we can perform the Calderón-Zygmund decomposition (for $|f|$ ) and so there exists a collection of balls $\left\{B_{i}\right\}$ such that $\left\{x \in \mathcal{X}: \mathcal{M} f(x)>\lambda C_{\mathcal{X}}\right\}=\bigcup_{i} B_{i}$. As in [CW] we decompose $f$ as $f=g+b=g+\sum_{i} b_{i}$, where

$$
\begin{aligned}
g(x) & =f(x) \chi_{\mathcal{X} \backslash \bigcup_{i} B_{i}}+\sum_{i}\left(\frac{1}{\mu\left(B_{i}\right)} \int_{B_{i}} f(y) \varrho_{i}(y) d \mu(y)\right) \chi_{B_{i}}(x), \\
b_{i}(x) & =f(x) \varrho_{i}(x)-\left(\frac{1}{\mu\left(B_{i}\right)} \int_{B_{i}} f(y) \varrho_{i}(y) d \mu(y)\right) \chi_{B_{i}}(x), \\
\varrho_{i}(x) & =\frac{\chi_{B_{i}(x)}}{\sum_{j} \chi_{B_{j}(x)}} \chi_{\bigcup_{j} B_{j} .}
\end{aligned}
$$

Let us note that by (iv) of Theorem 2.1, in each of the series above there are at most $M$ non-zero terms. This result and Remark 2.2 produce the following:

(A) If $x \in B_{i}$, then $M^{-1} \leq \varrho_{i}(x) \leq 1$. Moreover, $\sum_{i} \varrho_{i}(x)=\chi_{\bigcup_{i} B_{i}}(x)$.

(B) $|g(x)| \leq C \lambda$ for $\mu$-almost all $x \in \mathcal{X}$.

(C) $\operatorname{supp} b_{i} \subset B_{i}$ and $\frac{1}{\mu\left(B_{i}\right)} \int_{B_{i}}\left|b_{i}(x)\right| d \mu(x) \leq C \lambda$.

Then

$$
\begin{aligned}
& w\{x \in \mathcal{X}:|T f(x)|>\lambda\} \\
& \quad \leq w\left\{x \in \mathcal{X}:|T g(x)|>\frac{\lambda}{2}\right\}+w\left\{x \in \mathcal{X}:|T b(x)|>\frac{\lambda}{2}\right\} .
\end{aligned}
$$

Since $w \in \mathcal{A}_{1} \subset \mathcal{A}_{2}$, it follows that $T$ is a bounded operator on $L^{2}(\mathcal{X}, w)$. Then (B) yields

$$
\begin{aligned}
w\{x \in \mathcal{X}: \mid T g & \left.(x) \mid>\frac{\lambda}{2}\right\} \\
& \leq \frac{C}{\lambda^{2}} \int_{\mathcal{X}}|g(x)|^{2} w(x) d \mu(x) \leq \frac{C}{\lambda} \int_{\mathcal{X}}|g(x)| w(x) d \mu(x) \\
& \leq \frac{C}{\lambda}\left(\|f\|_{L^{1}(\mathcal{X}, w)}+\int_{\bigcup_{i} B_{i}}|g(x)| w(x) d \mu(x)\right) \\
& \leq \frac{C}{\lambda}\|f\|_{L^{1}(\mathcal{X}, w)},
\end{aligned}
$$

where for the latter estimate we have used the fact that $w \in \mathcal{A}_{1}$ and $(\mathrm{A})$ : 


$$
\int_{\cup_{i} B_{i}}|g(x)| w(x) d \mu(x) \leq \sum_{i} \int_{B_{i}}|f(y)| \varrho_{i}(y) \frac{w\left(B_{i}\right)}{\mu\left(B_{i}\right)} d \mu(y) \leq C\|f\|_{L^{1}(\mathcal{X}, w)}
$$

Let us show what to do with $T b$. Set $t_{i}=r_{B_{i}}^{m}$ and write

$$
b(x)=\sum_{i} b_{i}(x)=\sum_{i}\left(A_{t_{i}} b_{i}(x)+\left(b_{i}(x)-A_{t_{i}} b_{i}(x)\right)\right) .
$$

We want to split this series in two terms, so we need to study its convergence. By using the ideas of [DM, Theorem 1], and by (C), it follows that

$$
\begin{aligned}
\left|A_{t_{i}} b_{i}(x)\right| & \leq \sup _{y \in B_{i}} h_{t_{i}}(x, y) \int_{B_{i}}\left|b_{i}(y)\right| d \mu(y) \leq C \lambda \mu\left(B_{i}\right) \inf _{y \in B_{i}} h_{\theta t_{i}}(x, y) \\
& \leq C \lambda \int_{\mathcal{X}} h_{\theta t_{i}}(x, \xi) \chi_{B_{i}}(\xi) d \mu(\xi) .
\end{aligned}
$$

If $0 \leq u \in L^{p}(\mathcal{X})$ for some $p \geq 1$, it can be shown that

$$
\int_{\mathcal{X}}\left(\sum_{i}\left|A_{t_{i}} b_{i}(x)\right|\right) u(x) d \mu(x) \leq C M \lambda \int_{\mathcal{X}} \mathcal{M} u(y) \chi_{\bigcup_{i} B_{i}}(y) d \mu(y),
$$

where we have used (7) and Theorem 2.1. If we take $u \in L^{2}(\mathcal{X})$ with $\|u\|_{L^{2}(\mathcal{X})}=1$, then

$$
\begin{aligned}
\int_{\mathcal{X}}\left(\sum_{i}\left|A_{t_{i}} b_{i}(x)\right|\right) u(x) d \mu(x) & \leq C \lambda\|\mathcal{M} u\|_{L^{2}(\mathcal{X})}\left\|\chi_{\bigcup_{i} B_{i}}\right\|_{L^{2}(\mathcal{X})} \\
& \leq C \lambda^{1 / 2}\|f\|_{L^{1}(\mathcal{X})}^{1 / 2}
\end{aligned}
$$

by Theorem 2.1(iii). By taking the supremum over all these functions $u$, it follows that $\sum_{i}\left|A_{t_{i}} b_{i}\right| \in L^{2}(\mathcal{X})$ and $\sum_{i} A_{t_{i}} b_{i}$ is a Cauchy series in this space. It follows that $\sum_{i}\left(b_{i}-A_{t_{i}} b_{i}\right)$ also converges in $L^{2}(\mathcal{X})$. Since $T$ is continuous $T\left(\sum_{i}\left(b_{i}-A_{t_{i}} b_{i}\right)\right)=\sum_{i} T\left(b_{i}-A_{t_{i}} b_{i}\right)$ in $L^{2}(\mathcal{X})$ and consequently,

$$
\left|T\left(\sum_{i}\left(b_{i}-A_{t_{i}} b_{i}\right)\right)(x)\right| \leq \sum_{i}\left|T\left(b_{i}-A_{t_{i}} b_{i}\right)(x)\right|
$$

for $\mu$-almost every $x \in \mathcal{X}$. Then it is clear that

$$
\begin{aligned}
w\left\{x \in \mathcal{X}:|T b(x)|>\frac{\lambda}{2}\right\} \leq & w\left\{x \in \mathcal{X}:\left|T\left(\sum_{i} A_{t_{i}} b_{i}\right)(x)\right|>\frac{\lambda}{4}\right\} \\
& +w\left\{x \in \mathcal{X}:\left|T\left(\sum_{i}\left(b_{i}-A_{t_{i}} b_{i}\right)\right)(x)\right|>\frac{\lambda}{4}\right\} .
\end{aligned}
$$

We again use the fact that $T$ is bounded on $L^{2}(w)$ :

$$
\begin{aligned}
w\{x & \left.\in \mathcal{X}:\left|T\left(\sum_{i} A_{t_{i}} b_{i}\right)(x)\right|>\frac{\lambda}{4}\right\} \\
& \leq \frac{4}{\lambda^{2}} \int_{\mathcal{X}}\left|T\left(\sum_{i} A_{t_{i}} b_{i}\right)(x)\right|^{2} w(x) d \mu(x) \leq \frac{C}{\lambda^{2}}\left\|\sum_{i}\left|A_{t_{i}} b_{i}\right| \cdot w^{1 / 2}\right\|_{L^{2}(\mathcal{X})}^{2}
\end{aligned}
$$


We consider $0 \leq u \in L_{0}^{\infty}(\mathcal{X})$ with $\|u\|_{L^{2}(\mathcal{X})}=1$. We apply (15) to $u \cdot w^{1 / 2}$ (which belongs to $L^{2}(\mathcal{X})$, since $w$ is a locally integrable function) and we get

$$
\begin{aligned}
\int_{\mathcal{X}}\left(\sum_{i}\left|A_{t_{i}} b_{i}(x)\right| \cdot w(x)^{1 / 2}\right) u(x) d \mu(x) & \\
\leq & C \lambda \int_{\mathcal{X}} \mathcal{M}\left(u \cdot w^{1 / 2}\right)(y) \chi_{\bigcup_{i} B_{i}}(y) d \mu(y) \\
& \leq C \lambda\left\|\mathcal{M}\left(u \cdot w^{1 / 2}\right)\right\|_{L^{2}\left(\mathcal{X}, w^{-1}\right)}\left\|\chi_{\bigcup_{i} B_{i}}\right\|_{L^{2}(\mathcal{X}, w)} .
\end{aligned}
$$

Remark 2.2(i) and the fact that $w \in \mathcal{A}_{1}$ imply

$$
\left\|\chi_{\bigcup_{i} B_{i}}\right\|_{L^{2}(\mathcal{X}, w)}^{2}=w\left\{x \in \mathcal{X}: \mathcal{M} f(x)>\lambda C_{\mathcal{X}}\right\} \leq \frac{C}{\lambda}\|f\|_{L^{1}(\mathcal{X}, w)} .
$$

Moreover, since $w \in \mathcal{A}_{1}$, we have $w \in \mathcal{A}_{2}$ and thus $w^{-1} \in \mathcal{A}_{2}$, which implies that $\mathcal{M}$ is bounded on $L^{2}\left(\mathcal{X}, w^{-1}\right)$. Consequently,

$$
\int_{\mathcal{X}}\left(\sum_{i}\left|A_{t_{i}} b_{i}(x)\right| \cdot w(x)^{1 / 2}\right) u(x) d \mu(x) \leq C \lambda^{1 / 2}\|f\|_{L^{1}(\mathcal{X}, w)}^{1 / 2}
$$

for every $0 \leq u \in L_{0}^{\infty}(\mathcal{X})$ with $\|u\|_{L^{2}(\mathcal{X})}=1$. By taking the supremum over all these functions we eventually get

$$
w\left\{x \in \mathcal{X}:\left|T\left(\sum_{i} A_{t_{i}} b_{i}\right)(x)\right|>\frac{\lambda}{4}\right\} \leq \frac{C}{\lambda}\|f\|_{L^{1}(\mathcal{X}, w)} .
$$

On the other hand, set $\widetilde{B}_{i}=\left(1+c_{1}\right) B_{i}$ where $c_{1}$ is the constant in (b)'. Then, by using (16) and the fact that $w \in \mathcal{A}_{1}$ is a doubling measure we obtain

$$
\begin{aligned}
w\{x \in & \left.\mathcal{X}:\left|T\left(\sum_{i}\left(b_{i}-A_{t_{i}} b_{i}\right)\right)(x)\right|>\frac{\lambda}{4}\right\} \\
& \leq w\left(\bigcup_{i} \widetilde{B}_{i}\right)+w\left\{x \notin \bigcup_{i} \widetilde{B}_{i}:\left|T\left(\sum_{i}\left(b_{i}-A_{t_{i}} b_{i}\right)\right)(x)\right|>\frac{\lambda}{4}\right\} \\
& \leq C \sum_{i} w\left(B_{i}\right)+\frac{4}{\lambda} \sum_{i} \int_{\mathcal{X} \backslash \widetilde{B}_{i}}\left|T\left(b_{i}-A_{t_{i}} b_{i}\right)(x)\right| w(x) d \mu(x) .
\end{aligned}
$$

For the first term we use Theorem 2.1 and, as we did in (17), we obtain

$$
\begin{aligned}
\sum_{i} w\left(B_{i}\right) & =\int_{\mathcal{X}} \sum_{i} \chi_{B_{i}}(x) w(x) d \mu(x) \\
& \leq M w\left(\bigcup_{i} B_{i}\right) \leq \frac{C}{\lambda}\|f\|_{L^{1}(\mathcal{X}, w)} .
\end{aligned}
$$


On the other hand, by Remark 5.9 with $g=w \in L_{\text {loc }}^{1}(\mathcal{X})$,

$$
\begin{aligned}
\int_{\mathcal{X} \backslash \widetilde{B}_{i}}\left|T\left(b_{i}-A_{t_{i}} b_{i}\right)(x)\right| w(x) d \mu(x) \\
=\int_{\mathcal{X} \backslash \widetilde{B}_{i}}\left|\left(T-T A_{t_{i}}\right) b_{i}(x)\right| w(x) d \mu(x) \\
\leq C \int_{B_{i}}\left|b_{i}(y)\right| \int_{d(x, y) \geq c_{1} t_{i}^{1 / m}}\left|K(x, y)-k_{t_{i}}(x, y)\right| w(x) d \mu(x) d \mu(y) \\
\leq C \int_{B_{i}}\left|b_{i}(y)\right| \mathcal{M} w(y) d \mu(y) \leq C \int_{B_{i}}\left|b_{i}(y)\right| w(y) d \mu(y) \\
\leq C \int_{B_{i}}|f(y)| w(y) d \mu(y)+C \lambda w\left(B_{i}\right),
\end{aligned}
$$

where we used the fact that $w \in \mathcal{A}_{1}$ and Theorem 2.1. In this way, by (iv) of Theorem 2.1 and (19),

$$
\begin{aligned}
\sum_{i} \int_{\mathcal{X} \backslash \widetilde{B}_{i}} \mid & T\left(b_{i}-A_{t_{i}} b_{i}\right)(x) \mid w(x) d \mu(x) \\
& \leq C \sum_{i} \int_{B_{i}}|f(y)| w(y) d \mu(y)+C \lambda \sum_{i} w\left(B_{i}\right) \leq C\|f\|_{L^{1}(\mathcal{X}, w)},
\end{aligned}
$$

which allows us to get

$$
w\left\{x \in \mathcal{X}:\left|T\left(\sum_{i}\left(b_{i}-A_{t_{i}} b_{i}\right)\right)(x)\right|>\frac{\lambda}{4}\right\} \leq \frac{C}{\lambda}\|f\|_{L^{1}(\mathcal{X}, w)} .
$$

For every $\lambda>\|f\|_{L^{1}(\mathcal{X})}(\mu(\mathcal{X}))^{-1}$, this estimate, (14), and (18) lead to

$$
w\{x \in \mathcal{X}:|T f(x)|>\lambda\} \leq \frac{C}{\lambda}\|f\|_{L^{1}(\mathcal{X}, w)} .
$$

If $\mathcal{X}$ is unbounded, the proof is complete because the former inequality holds for every $\lambda>0$. Otherwise, we have to consider what happens for $0<\lambda \leq\|f\|_{L^{1}(\mathcal{X})}(\mu(\mathcal{X}))^{-1}$. Since $\mathcal{X}$ is bounded we can write $\mathcal{X}=B\left(x_{0}, r_{0}\right)$. Then, as $w \in \mathcal{A}_{1}$, we conclude

$$
\begin{aligned}
w\{x \in \mathcal{X}:|T f(x)|>\lambda\} & \leq w(\mathcal{X}) \leq \frac{1}{\lambda} \int_{\mathcal{X}}|f(x)| \frac{w(\mathcal{X})}{\mu(\mathcal{X})} d \mu(x) \\
& \leq \frac{C}{\lambda}\|f\|_{L^{1}(\mathcal{X}, w)} .
\end{aligned}
$$

6. Singular integral operators on irregular domains. In what follows, $\Omega$ will be a measurable subset of the space of homogeneous type $(\mathcal{X}, d, \mu)$. It is well known that under certain regularity assumptions on $\Omega$, for instance Lipschitz boundary, $\Omega$ with the restriction of $\mu$ is itself a space 
of homogeneous type. Thus, the previous results can be applied directly to get boundedness of singular operators with Muckenhoupt weights defined in $\Omega$. However, there are interesting problems where the natural domains are not smooth, for example open subsets of the Euclidean space $\mathbb{R}^{d}$. This is the case of boundary value problems for partial differential equations. In those problems, the restriction of the measure might fail to satisfy the doubling property and therefore $\Omega$ would not be a space of homogeneous type.

We are interested in dealing with weighted norm estimates in those contexts. As pointed out in $[\mathrm{DM}]$, one can extend the singular operators defined in $\Omega$ to the space $\mathcal{X}$. Since there is no assumption on the regularity of the kernels in the space variables, the extension of the kernel still satisfies similar conditions. Given $T$, a bounded linear operator on $L^{p}(\Omega)$, $1<p<\infty$, the extension of $T$ to $\mathcal{X}$ is defined as $\widetilde{T} f(x)=T\left(f \chi_{\Omega}\right)(x) \chi_{\Omega}(x)$ for $f \in L^{p}(\mathcal{X})$. Then $T$ is bounded on $L^{p}(\Omega)$ if and only if $\widetilde{T}$ is bounded on $L^{p}(\mathcal{X})$. In a similar way, there is an equivalence between weak type estimates. If $K$ is the kernel of $T$, then the associated kernel of $\widetilde{T}$ is given by $\widetilde{K}(x, y)=K(x, y) \chi_{\Omega \times \Omega}(x, y)$. As observed in $[\mathrm{DM}]$, it is easy to see that a Hörmander condition for $K$ would not necessarily imply such a condition for $\widetilde{K}$ because this kernel is even discontinuous. Nevertheless, since the conditions assumed on the kernels do not involve their regularity, they imply similar properties of the kernels of the extended operators.

We are going to use the following notation: $B^{\mathcal{X}}$ and $B^{\Omega}$ denote respectively balls in $\mathcal{X}$ and $\Omega$. The "approximations of the identity" $\left\{D_{t}: t>0\right\}$ in this context are given by

$$
D_{t} f(x)=\int_{\mathcal{X}} a_{t}(x, y) f(y) d \mu(y), \quad f \in L^{p}(\Omega) \text { for some } p \geq 1 .
$$

The kernels are assumed to satisfy $\left|a_{t}(x, y)\right| \leq h_{t}(x, y)$ for every $x, y \in \Omega$, where $h_{t}(x, y)$ is defined in $\mathcal{X} \times \mathcal{X}$ as

$$
h_{t}(x, y)=\frac{1}{\mu\left(B^{\mathcal{X}}\left(x, t^{1 / m}\right)\right)} s\left(d(x, y)^{m} t^{-1}\right)
$$

Again, $m$ is a positive constant and $s$ is a positive, bounded, decreasing function satisfying (4). The hypotheses on the operators are:

(a) $\Omega T$ is a bounded linear operator from $L^{2}(\Omega)$ to $L^{2}(\Omega)$ with kernel $K$ such that, for every $f \in L_{0}^{\infty}(\Omega)$,

$$
T f(x)=\int_{\mathcal{X}} K(x, y) f(y) d \mu(y) \quad \text { for } \mu \text {-almost all } x \notin \operatorname{supp} f .
$$

(b) $\Omega$ There exists an "approximation of the identity" $\left\{A_{t}: t>0\right\}$ such that $T A_{t}$ has associated kernel $k_{t}(x, y)$ and there are $c_{1}, c_{2}>0$ such that

$$
\int_{d(x, y) \geq c_{1} t^{1 / m}}\left|K(x, y)-k_{t}(x, y)\right| d \mu(x) \leq c_{2} \quad \text { for all } y \in \Omega .
$$


(c) $\Omega$ There exists an "approximation of the identity" $\left\{D_{t}: t>0\right\}$ such that $D_{t} T$ has kernel $K_{t}(x, y)$ and, for some constants $c_{3}, c_{4}, \alpha>0$,

$$
\begin{aligned}
& (\mathrm{c} 1)_{\Omega}\left|K_{t}(x, y)\right| \leq c_{4} \frac{1}{\mu\left(B^{\mathcal{X}}\left(x, t^{1 / m}\right)\right)} \text { for } x, y \in \Omega \text { with } d(x, y) \leq c_{3} t^{1 / m}, \\
& (\mathrm{c} 2)_{\Omega}\left|K_{t}(x, y)-K(x, y)\right| \leq c_{4} \frac{1}{\mu\left(B^{\mathcal{X}}(x, d(x, y))\right)} \frac{t^{\alpha / m}}{d(x, y)^{\alpha}} \\
& \quad \text { for } x, y \in \Omega \text { with } d(x, y) \geq c_{3} t^{1 / m} .
\end{aligned}
$$

In $[\mathrm{DM}]$ it is proved that if $T$ satisfies $(\mathrm{a})_{\Omega}$ and $(\mathrm{b})_{\Omega}$, then it is of weak type $(1,1)$ in $\Omega$ and bounded on $L^{p}(\Omega)$ for $1<p \leq 2$. Assuming additionally (c) $\Omega$, the authors obtain the complete range $1<p<\infty$ and the fact that the associated maximal operator $T_{\star}$ is also continuous on $L^{p}(\Omega), 1<p<\infty$. The way of proving these estimates is based on extending all the operators involved to $\mathcal{X}$. That is, they consider $\widetilde{T}, \widetilde{A}_{t}$ and $\widetilde{D}_{t}$ and they prove that (a) $\Omega,(\mathrm{b})_{\Omega}$ and (c) $\Omega$ imply that $\widetilde{T}$ satisfies (a), (b) and (c). Then they just apply the obtained results in $\mathcal{X}$ to get the desired estimates.

By using those ideas, we can now state that these operators also satisfy weighted norm inequalities. We just need to apply Theorem 5.3, Corollary 5.7, and Theorem 5.8 to the extended operator $\widetilde{T}$ to find that $T$ and $T_{\star}$ satisfy weighted estimates for Muckenhoupt weights defined in $\mathcal{X}$. We use the notation $\mathcal{A}_{p}(\mathcal{X})$ to make it clear that the Muckenhoupt weights are considered in the whole space $\mathcal{X}$.

TheORem 6.1. Let $T$ be an operator satisfying $(\mathrm{a})_{\Omega},(\mathrm{b})_{\Omega}$ and $(\mathrm{c})_{\Omega}$.

(i) If $w \in \mathcal{A}_{p}(\mathcal{X}), 1<p<\infty$, then $T$ and $T_{\star}$ are bounded on $L^{p}(\Omega, w)$.

(ii) Replace $(\mathrm{b})_{\Omega}$ by the following stronger condition:

$(\mathrm{b})_{\Omega}^{\prime}$ There exists an "approximation of the identity" $\left\{A_{t}: t>0\right\}$ such that the operators $T A_{t}$ have associated kernels $k_{t}(x, y)$ and there exist $c_{1}, c_{2}, \beta>0$ such that

$$
\left|K(x, y)-k_{t}(x, y)\right| \leq c_{2} \frac{1}{\mu\left(B^{\mathcal{X}}(y, d(x, y))\right)} \frac{t^{\beta / m}}{d(x, y)^{\beta}}
$$

for all $x, y \in \Omega$ with $d(x, y) \geq c_{1} t^{1 / m}$.

Then, for every $w \in \mathcal{A}_{1}(\mathcal{X}), T$ maps $L^{1}(\Omega, w)$ into $L^{1, \infty}(\Omega, w)$.

7. Holomorphic functional calculi of elliptic operators. We are going to review some of the necessary background. For a complete account the reader is referred to $[\mathrm{ADM}]$ or also to $[\mathrm{DM}]$. Given $\theta$ and $\nu$ with $0<$ $\theta<\nu<\pi$ we define

$$
S_{\theta}=\{\zeta \in \mathbb{C}:|\arg (\zeta)| \leq \theta\} \cup\{0\} .
$$


and its interior is denoted by $S_{\theta}^{0}$. Let $H\left(S_{\nu}^{0}\right)$ be the space of holomorphic functions defined in $S_{\nu}^{0}$. We are going to consider the following subspaces of $H\left(S_{\nu}^{0}\right)$ :

$$
\begin{aligned}
H^{\infty}\left(S_{\nu}^{0}\right) & =\left\{f \in H\left(S_{\nu}^{0}\right):\|f\|_{\infty}<\infty\right\}, \quad \text { where }\|f\|_{\infty}=\sup \left\{|f(\zeta)|: \zeta \in S_{\nu}^{0}\right\} \\
\Psi\left(S_{\nu}^{0}\right) & =\left\{\psi \in H\left(S_{\nu}^{0}\right): \exists s>0,|\psi(\zeta)| \leq C|\zeta|^{s}\left(1+|\zeta|^{2 s}\right)^{-1}\right\} \\
F\left(S_{\nu}^{0}\right) & =\left\{f \in H\left(S_{\nu}^{0}\right): \exists s>0,|f(\zeta)| \leq C\left(|\zeta|^{-s}+|\zeta|^{s}\right)\right\}
\end{aligned}
$$

It is clear that $\Psi\left(S_{\nu}^{0}\right) \subset H^{\infty}\left(S_{\nu}^{0}\right) \subset F\left(S_{\nu}^{0}\right)$. A closed operator $L$ in some Banach space $\mathbb{A}$ is said to be of type $\theta, 0 \leq \theta<\pi$, if its spectrum $\sigma(L) \subset S_{\theta}$ and for every $\nu>\theta$, there exists $C_{\nu}$ such that

$$
\left\|(L-\zeta I)^{-1}\right\| \leq C_{\nu}|\zeta|^{-1}, \quad \zeta \notin S_{\nu} .
$$

By the Hille-Yosida theorem, such an operator with $\theta<\pi / 2$ is the generator of a bounded holomorphic semigroup $e^{-z L}$ in the sector $S_{\nu}^{0}$ with $\nu=\pi / 2-\theta$.

Let us assume that $L$ is a one-one operator of type $\theta$ with dense domain and dense range on $\mathbb{A}$. We can define a holomorphic calculus in the following way: if $\psi \in \Psi\left(S_{\nu}^{0}\right)$, then

$$
\psi(L)=\frac{1}{2 \pi i} \int_{\gamma}(L-\zeta I)^{-1} \psi(\zeta) d \zeta
$$

where, for $\theta<\eta<\nu, \gamma$ is the contour $\left\{\zeta=r e^{ \pm i \eta}: r \geq 0\right\}$ parameterized clockwise around $S_{\theta}$. This integral is absolutely convergent in $\mathcal{L}(\mathbb{A})$. Moreover, by the Cauchy theorem this definition does not depend on the contour, that is, is independent of the choice of $\eta \in(\theta, \nu)$. If $f \in F\left(S_{\nu}^{0}\right)$ with $|f(\zeta)| \leq C\left(|\zeta|^{k}+|\zeta|^{-k}\right)$ for $\zeta \in S_{\nu}^{0}$, we take

$$
\psi(\zeta)=\left(\frac{\zeta}{(1+\zeta)^{2}}\right)^{k+1} .
$$

Then $\psi, \psi f \in \Psi\left(S_{\nu}^{0}\right)$ and $\psi(L)$ is one-one. In this way, the operator $(f \psi)(L)$ is bounded and $\psi(L)^{-1}$ is a closed operator in $\mathbb{A}$. Then we define $f(L)=$ $\psi(L)^{-1}(f \psi)(L)$.

A very useful result about holomorphic functional calculi is the following convergence lemma obtained by McIntosh in [McI]; for a proof the reader is also referred to $[\mathrm{ADM}$, Theorem D].

Lemma 7.1 ([McI]). Let $0 \leq \theta<\nu \leq \pi$. Let $L$ be an operator of type $\theta$ which is one-one with dense domain and range on $\mathbb{A}$. Let $\left\{f_{\alpha}\right\}$ be a uniformly bounded net in $H^{\infty}\left(S_{\nu}^{0}\right)$ which converges to $f \in H^{\infty}\left(S_{\nu}^{0}\right)$ uniformly on compact subsets of $S_{\nu}^{0}$, such that $\left\{f_{\alpha}(L)\right\}$ is a uniformly bounded net in $\mathcal{L}(\mathbb{A})$. Then $f(L) \in \mathcal{L}(\mathbb{A}), f_{\alpha}(L) u \rightarrow f(L) u$ for all $u \in \mathbb{A}$, and $\|f(L)\| \leq$ $\sup _{\alpha}\left\|f_{\alpha}(L)\right\|$. 
Coming back to our setting, let us suppose that $\Omega$ is a measurable subset of a space of homogeneous type $(\mathcal{X}, d, \mu)$. Let $L$ be an operator of type $\theta<\pi / 2$ on $L^{2}(\Omega)$ so that $-L$ generates a holomorphic semigroup $e^{-z L}$, $|\arg (z)|<\pi / 2-\theta$.

TheOrem 7.2 ([DM, Theorem 6]). Assume the following conditions:

(a) The holomorphic semigroup $e^{-z L},|\arg (z)|<\pi / 2-\theta$, is represented by the kernels $a_{z}(x, y)$ which satisfy, for all $\nu>\theta$, an estimate

$$
\left|a_{z}(x, y)\right| \leq C_{\nu} h_{|z|}(x, y) \quad \text { for } x, y \in \Omega \text { and }|\arg (z)|<\pi / 2-\nu,
$$

where $h_{t}$ is defined on $\mathcal{X} \times \mathcal{X}$ as in $(20)$.

(b) The operator $L$ has a bounded functional calculus in $L^{2}(\Omega)$. That is, for any $\nu>\theta$ and $f \in H^{\infty}\left(S_{\nu}^{0}\right)$, the operator $f(L)$ satisfies $\|f(L)\|_{L^{2}(\Omega) \rightarrow L^{2}(\Omega)}$ $\leq C_{\nu}\|f\|_{\infty}$.

Then the operator $L$ has a bounded functional calculus in $L^{p}(\Omega)$, $1<p<\infty$, that is, for all $f \in H^{\infty}\left(S_{\nu}^{0}\right)$, we have $\|f(L)\|_{L^{p}(\Omega) \rightarrow L^{p}(\Omega)} \leq$ $C_{p, \nu}\|f\|_{\infty}$. When $p=1$, the operator $f(L)$ is of weak type $(1,1)$. Furthermore, if we define $T=f(L)$, then the maximal truncated operator $T_{\star}$ is bounded on $L^{p}(\Omega)$ for all $p, 1<p<\infty$.

The proof of this result goes as follows. First, the authors consider the case $T=f(L)$ with $f \in \Psi\left(S_{\nu}^{0}\right), \nu>\theta$. For such an operator, (a) $\Omega,(\mathrm{b})_{\Omega}$ and (c) $\Omega$ are obtained by taking as "approximations of the identity" $A_{t}=D_{t}$ $=e^{-t L}$. Then McIntosh's convergence lemma (Lemma 7.1 above) allows them to extend the result to $H^{\infty}\left(S_{\nu}^{0}\right)$.

Taking into account that proof and the fact that the required conditions for the weighted inequalities are the same as those assumed in $[\mathrm{DM}]$, we can state the following result just by using Theorem 6.1. Let us note that in part (ii) we added a stronger condition (b) ${ }_{\Omega}^{\prime}$ which can be checked easily: the commutation property of the functional calculus and the fact that $A_{t}=$ $D_{t}=e^{-t L}$ yield $K_{t}=k_{t}$. Moreover, by (3), we see that $\mu\left(B^{\mathcal{X}}(y, d(x, y))\right)$ and $\mu\left(B^{\mathcal{X}}(x, d(x, y))\right)$ are comparable and hence $(\mathrm{b})_{\Omega}^{\prime}$ arises from $(\mathrm{c} 2)_{\Omega}$ by taking $\beta=\alpha$.

THEOREM 7.3. Under the conditions of the previous theorem, for any $1<p<\infty$ and $w \in \mathcal{A}_{p}(\mathcal{X})$, the operator $L$ has a bounded holomorphic functional calculus in $L^{p}(\Omega, w)$. That is, if $\nu>\theta$ and $f \in H^{\infty}\left(S_{\nu}^{0}\right)$, then

$$
\|f(L)\|_{L^{p}(\Omega, w) \rightarrow L^{p}(\Omega, w)} \leq C_{p, w, \nu}\|f\|_{\infty} .
$$

Furthermore, when $p=1, f(L)$ is bounded from $L^{1}(\Omega, w)$ to $L^{1, \infty}(\Omega, w)$ for every $w \in \mathcal{A}_{1}(\mathcal{X})$. In addition, if we write $T=f(L)$, then $T_{\star}$ is bounded from $L^{p}(\Omega, w)$ to $L^{p}(\Omega, w)$ for any $1<p<\infty$ and $w \in \mathcal{A}_{p}(\mathcal{X})$. 
We are going to present some applications for this result. We work with some operators taken from [AE] which correspond to second order elliptic operators with different boundary conditions. In what follows, our setting will be $\mathbb{R}^{d}$ with the Euclidean distance and the Lebesgue measure. Besides, $\Omega$ will be an open subset of this space of homogeneous type. For $1 \leq p \leq \infty$, the Sobolev space $W^{1, p}(\Omega)$ consists of all functions in $L^{p}(\Omega)$ with first order distributional partial derivatives in the same space. As usual, when $p=2$, we write $H^{1}(\Omega)$ instead of $W^{1,2}(\Omega)$. Finally, $H_{0}^{1}(\Omega)$ is the closure in $H^{1}(\Omega)$ of $C_{0}^{\infty}(\Omega)$.

We are going to consider second order elliptic operators with the following boundary conditions (for more details see $[\mathrm{AE}]$ ):

(A) Dirichlet boundary conditions. Let $a_{i j} \in W^{1, \infty}(\Omega)$ be real functions for $i, j=1, \ldots, d$; let $b_{i}, c_{i} \in W^{1, \infty}(\Omega), i=1, \ldots, d$ (complex) and let $c_{0} \in L^{\infty}(\Omega)$. We consider the sesquilinear form $a: H_{0}^{1}(\Omega) \times H_{0}^{1}(\Omega) \rightarrow \mathbb{C}$ defined by

$$
a(u, v)=\sum_{i, j=1}^{d} \int_{\Omega} a_{i j} D_{i} u \overline{D_{j} v}+\sum_{i=1}^{d} \int_{\Omega} b_{i} D_{i} u \bar{v}+\sum_{i=1}^{d} \int_{\Omega} c_{i} u \overline{D_{i} v}+\int_{\Omega} c_{0} u \bar{v} .
$$

Suppose that there exists $\delta>0$ such that the following ellipticity condition holds:

$$
\sum_{i, j=1}^{d} a_{i j}(x) \xi_{i} \xi_{j} \geq \delta|\xi|^{2} \quad \text { for all } \xi \in \mathbb{R}^{d} \text { and almost all } x \in \Omega .
$$

Then $A$ is the operator associated with $a$ and generates a semigroup $\left(e^{-t A}\right)_{t>0}$.

(B) General boundary conditions. We consider operators as before but now the coercive form is defined in a certain domain $V \times V$. Some conditions are imposed on this domain in order to obtain Gaussian estimates for the kernel of the semigroup. Now, all functions are real-valued, so we only work in the real field. Assume that $V$ satisfies:

(i) $V$ is a closed subset of $H^{1}(\Omega)$ and $H_{0}^{1}(\Omega) \subset V$.

(ii) $V$ has the $L^{1}-H^{1}$ extension property, that is, there exists a continuous linear operator $\mathcal{E}: V \rightarrow H^{1}\left(\mathbb{R}^{d}\right)$, called an extension operator, such that $\left.(\mathcal{E} \varphi)\right|_{\Omega}=\varphi$ for all $\varphi \in V$, and

$$
\|\mathcal{E} \varphi\|_{L^{1}\left(\mathbb{R}^{d}\right)} \leq C\|\varphi\|_{L^{1}(\Omega)} \quad \text { for all } \varphi \in V \cap L^{1}(\Omega) .
$$

For instance, if $V=H_{0}^{1}(\Omega)$, this operator extends functions on $\Omega$ by 0 on $\mathbb{R}^{d} \backslash \Omega$ (see [AE] for more examples).

(iii) If $v \in V$ then $|v|, \min \{|v|, 1\} \in V$.

(iv) If $v \in V, u \in H^{1}(\Omega)$ with $|u| \leq v$, then $u \in V$ ( $V$ is an ideal in $\left.H^{1}(\Omega)\right)$. 
Let us consider the bilinear form $a: V \times V \rightarrow \mathbb{R}$ given by

$$
a(u, v)=\sum_{i, j=1}^{d} \int_{\Omega} a_{i j} D_{i} u D_{j} v+\sum_{i=1}^{d} \int_{\Omega} b_{i} D_{i} u v+\sum_{i=1}^{d} \int_{\Omega} c_{i} u D_{i} v+\int_{\Omega} c_{0} u v,
$$

and assume the ellipticity condition (21). Now, all coefficients are real-valued and it is supposed that $a_{i j} \in L^{\infty}(\Omega), b_{i}, c_{i} \in W^{1, \infty}(\Omega), c_{0} \in L^{\infty}(\Omega)$. The first order coefficients are required to satisfy:

$$
\text { If } v \in V \text {, then } b_{i} v, c_{i} v \in H_{0}^{1}(\Omega) \text { for all } i=1, \ldots, d \text {. }
$$

Under these hypotheses $A$ is the operator associated to the coercive form $a$ and generates a positive semigroup $\left(e^{-t A}\right)_{t>0}$. For example, if $V=H_{0}^{1}(\Omega)$, we are considering an elliptic operator with Dirichlet boundary conditions and if $b_{i}, c_{i} \in W^{1, \infty}(\Omega)$, then (22) holds. On the other hand, by taking $V=$ $H^{1}(\Omega)$ with $\partial \Omega$ minimally smooth, the problem has Neumann boundary conditions. In this case, (22) holds provided that $b_{i}, c_{i} \in W_{0}^{1, \infty}(\Omega)$. If $\Omega$ is bounded, then $b_{i}, c_{i} \in H_{0}^{1}(\Omega)$ is a necessary condition for (22), since $1 \in V$.

(C) Robin boundary conditions. Again we work in the real field and $\Omega$ is a bounded open set in $\mathbb{R}^{d}$ with Lipschitz boundary $\Gamma=\partial \Omega$. We write $d \gamma$ for the restriction of the Lebesgue measure to $\Gamma$. There exists a unique linear bounded operator $\operatorname{tr}: H^{1}(\Omega) \rightarrow L^{2}(\Gamma, d \gamma)$ such that $\operatorname{tr} u=$ $\left.u\right|_{\Gamma}$ for all $u \in H^{1}(\Omega) \cap C(\bar{\Omega})$. This operator is called the trace operator. Assume that the domain $V$ satisfies conditions (i)-(iv) above. We consider the same form $a$ defined in the previous example with domain $V \times V$. We also assume (21), (22). Consider a bilinear form $b: V \times V \rightarrow \mathbb{R}$ given by

$$
b(u, v)=\int_{\Gamma} \beta(x)(\operatorname{tr} u)(x)(\operatorname{tr} v)(x) d \gamma(x),
$$

where $\beta \in L^{\infty}(\Gamma)$ is a positive function. Let us consider the coercive form $q=a+b$ with domain $V$. Then $A$ is the operator associated to $q$ and $A$ generates a positive semigroup $\left(e^{-t A}\right)_{t>0}$. Let us note that Robin boundary conditions coincide with Dirichlet boundary conditions if $V=H_{0}^{1}(\Omega)$ and with Neumann boundary conditions if $V=H^{1}(\Omega)$ and $\beta=0$.

We consider a second order elliptic operator $A$ as in (A), (B) or (C). Let $\left(e^{-t A}\right)_{t>0}$ be the generated semigroup. As in [AE], there exist $b, c>0, \nu \in \mathbb{R}$ such that $\left(e^{-t A}\right)_{t>0}$ is given by the kernels $a_{t} \in L^{\infty}(\Omega \times \Omega)$ which satisfy the following Gaussian estimate:

$$
\left|a_{t}(x, y)\right| \leq c t^{-d / 2} e^{-b|x-y|^{2} t^{-1}} e^{\nu t} \quad \text { for a.e. }(x, y) \in \Omega \times \Omega .
$$

In cases $(\mathrm{B})$ and $(\mathrm{C})$, the kernels also satisfy $a_{t}(x, y) \geq 0$ for almost all $(x, y) \in \Omega \times \Omega$. We complexify the domain $V$ and the coercive form $a$ in 
those cases. Set

$$
\theta_{a}=\frac{\pi}{2}-\inf \left\{\theta>0: \sum_{i, j=1}^{d} a_{i j}(x) \xi_{i} \bar{\xi}_{j} \in S_{\theta}^{0} \text { for all } \xi \in \mathbb{C}^{d} \text { and a.e. } x \in \Omega\right\} \text {. }
$$

Note that $\theta_{a}=\pi / 2$ if $\left(a_{i j}\right)_{i j}$ is a symmetric matrix for almost every $x \in \Omega$. Hence, $e^{-z A}$ is a holomorphic semigroup in $L^{2}(\Omega)$ with holomorphy sector which contains at least $S_{\theta_{a}}^{0}$. Moreover, let $\theta \in\left[0, \theta_{a}\right)$ and let $\lambda_{0} \in \mathbb{R}$ be such that

$$
\left\|e^{-z A}\right\|_{L^{2}(\Omega) \rightarrow L^{2}(\Omega)} \leq e^{\lambda_{0}|z|} \quad \text { for all } z \in S_{\theta}
$$

then, for all $\lambda>\lambda_{0}$, there exist $b, c>0$ such that the kernel of the semigroup satisfies $\left|a_{z}(x, y)\right| \leq c|z|^{-d / 2} e^{-b|x-y|^{2}|z|^{-1}} e^{\lambda|z|}$, for almost all $(x, y) \in \Omega \times \Omega$, uniformly on $z \in S_{\theta}$ (see [AE, Theorem 5.6]).

Theorem 7.4 ([AE, Theorem 5.9, Corollary 5.10]). Let us adopt the notation and the hypotheses of examples (A), (B) or (C). Let $\pi / 2-\theta_{a}<$ $\nu<\pi / 2$ and $\lambda_{0} \in \mathbb{R}$ be such that

$$
\left\|e^{-z A}\right\|_{L^{2}(\Omega) \rightarrow L^{2}(\Omega)} \leq e^{\lambda_{0}|z|} \quad \text { for all } z \in S_{\pi / 2-\nu} .
$$

Then for all $\lambda>\lambda_{0}$, the operator $A+\lambda I$ has a bounded holomorphic calculus in $L^{p}(\Omega)$ for every $1<p<\infty$. In particular,

$$
\left\|(A+\lambda I)^{i s}\right\|_{L^{p}(\Omega) \rightarrow L^{p}(\Omega)} \leq C e^{\nu|s|}
$$

uniformly for all $s \in \mathbb{R}$ and $1<p<\infty$. Moreover, for every $f \in H^{\infty}\left(S_{\nu}^{0}\right)$, the operator $f(A+\lambda I)$ is of weak type $(1,1)$.

The proof consists in showing that $\left|\widehat{a}_{z}(x, y)\right| \leq c|z|^{-d / 2} e^{-b|x-y|^{2}|z|^{-1}}$ for $z \in S_{\nu}^{0}$, where $\widehat{a}_{z}$ is the kernel of the semigroup $e^{-(A+\lambda I) z}$, and then using [AE, Theorem 5.7]. This result is known under the hypothesis that either $\partial \Omega$ is a null set in $\mathbb{R}^{d}$ or $\Omega=\mathbb{R}^{d}$. In general, for $\Omega$ an arbitrary open set, the authors point out that the proof in [DR] can be modified in order to cover this case. On the other hand, this result arises from Theorem 7.2 proved in $[\mathrm{DM}]$. Let us remark that $\left|\widehat{a}_{z}(x, y)\right| \leq h_{|z|}(x, y)$ where, for $t>0$ and $(x, y) \in \mathbb{R}^{d} \times \mathbb{R}^{d}$,

$$
h_{t}(x, y)=c|t|^{-d / 2} e^{-b|x-y|^{2}|t|^{-1}}=\frac{1}{\left|B\left(x, t^{1 / 2}\right)\right|} s\left(|x-y|^{2} t^{-1}\right)
$$

and $s(r)=c e^{-b r}$ is a positive, bounded, decreasing function. With respect to the notation in (20), $m=2$ and it is clear that (4) holds for every $\vartheta>0$. In other words, we are under the assumptions of Theorem 7.2 with $L=A+\lambda I$. As a consequence and simply by Theorem 7.3, we can prove weighted estimates and that $L$ has a bounded holomorphic calculus in weighted Lebesgue spaces. 
COROLlary 7.5. Let us adopt the notation and the hypotheses of examples (A), (B) or (C). Let $\pi / 2-\theta_{a}<\nu<\pi / 2$ and $\lambda_{0} \in \mathbb{R}$ be such that

$$
\left\|e^{-z A}\right\|_{L^{2}(\Omega) \rightarrow L^{2}(\Omega)} \leq e^{\lambda_{0}|z|} \quad \text { for all } z \in S_{\pi / 2-\nu} .
$$

Then, for all $\lambda>\lambda_{0}$, we have:

(i) For any $1<p<\infty$ and $w \in \mathcal{A}_{p}(\mathcal{X})$, the operator $A+\lambda I$ has a bounded holomorphic calculus in $L^{p}(\Omega, w)$, that is,

$$
\|f(A+\lambda I)\|_{L^{p}(\Omega, w) \rightarrow L^{p}(\Omega, w)} \leq C_{p, w, \nu}\|f\|_{\infty} \quad \text { for all } f \in H^{\infty}\left(S_{\nu}^{0}\right) .
$$

(ii) For any $w \in \mathcal{A}_{1}(\mathcal{X})$ and $f \in H^{\infty}\left(S_{\nu}^{0}\right)$ the operator $f(A+\lambda I)$ is bounded from $L^{1}(\Omega, w)$ to $L^{1, \infty}(\Omega, w)$.

(iii) If we write $T=f(A+\lambda I)$, where $f \in H^{\infty}\left(S_{\nu}^{0}\right)$, then $T_{\star}$ maps $L^{p}(\Omega, w)$ into $L^{p}(\Omega, w)$ for every $1<p<\infty$ and $w \in \mathcal{A}_{p}(\mathcal{X})$.

By taking $f(z)=z^{i s}$ with $s \in \mathbb{R}, T=(A+\lambda I)^{i s}$ satisfies all these estimates and, in particular, for $1<p<\infty, w \in \mathcal{A}_{p}(\mathcal{X})$ and $s \in \mathbb{R}$,

$$
\left\|(A+\lambda I)^{i s}\right\|_{L^{p}(\Omega, w) \rightarrow L^{p}(\Omega, w)} \leq C e^{\nu|s|} .
$$

\section{References}

[ADM] D. Albrecht, X. Duong and A. McIntosh, Operator theory and harmonic analysis, in: Instructional Workshop on Analysis and Geometry, Part III (Canberra, 1995), Proc. Centre Math. Appl. Austral. Nat. Univ. 34, Austral. Nat. Univ., Canberra, 1996, 77-136.

[AE] W. Arendt and A. F. M. ter Elst, Gaussian estimates for second order elliptic operators with boundary conditions, J. Operator Theory 38 (1997), 87-130.

[BG] D. L. Burkholder and R. F. Gundy, Distribution function inequalities for the area integral, Studia Math. 44 (1972), 527-544.

[CF] R. R. Coifman and C. Fefferman, Weighted norm inequalities for maximal functions and singular integrals, ibid. 51 (1974), 241-250.

[CW] R. R. Coifman et G. Weiss, Analyse harmonique non commutative sur certains espaces homogènes, Lecture Notes in Math. 242, Springer, 1971.

[DM] X. T. Duong and A. McIntosh, Singular integral operators with non-smooth kernel on irregular domains, Rev. Mat. Iberoamericana 15 (1999), 233-265.

[DR] X. T. Duong and D. W. Robinson, Semigroup kernels, Poisson bounds, and holomorphic functional calculus, J. Funct. Anal. 142 (1996), 89-128.

[DY] X. T. Duong and L. X. Yan, Weighted inequalities for holomorphic functional calculi of elliptic operators, preprint, 2001.

[FS] C. Fefferman and E. M. Stein, $H^{p}$ spaces of several variables, Acta Math. 129 (1972), 137-193.

[GM] J. García-Cuerva and J. M. Martell, Weighted inequalities and vector-valued Calderón-Zygmund operators on non-homogeneous spaces, Publ. Mat. 44 (2000), 613-640.

[GR] J. García-Cuerva and J. L. Rubio de Francia, Weighted Norm Inequalities and Related Topics, North-Holland Math. Stud. 116, North-Holland, 1985. 
[MS1] R. A. Macías and C. Segovia, Lipschitz functions on spaces of homogeneous type, Adv. Math. 33 (1979), 257-270.

[MS2] - - - A decomposition into atoms of distributions on spaces of homogeneous type, ibid., 271-309.

[Mar] J. M. Martell, Desigualdades con pesos en el Análisis de Fourier: de los espacios de tipo homogéneo a las medidas no doblantes, Ph.D. thesis, Universidad Autónoma de Madrid, 2001.

[McI] A. McIntosh, Operators which have an $H_{\infty}$ functional calculus, in: Miniconference on Operator Theory and Partial Differential Equations (North Ryde, 1986), Proc. Centre Math. Anal. Austral. Nat. Univ. 14, Austral. Nat. Univ., Canberra, 1986, 210-231.

[Muc] B. Muckenhoupt, Weighted norm inequalities for the Hardy maximal function, Trans. Amer. Math. Soc. 165 (1972), 207-226.

[ST] J.-O. Strömberg and A. Torchinsky, Weighted Hardy Spaces, Lecture Notes in Math. 1381, Springer, 1989.

Departamento de Matemáticas, C-XV

Universidad Autónoma de Madrid

28049 Madrid, Spain

E-mail: chema.martell@uam.es

Received June 10, 2002

Revised version July 16, 2003 